\title{
LIN28B alters ribosomal dynamics to promote metastasis in MYCN-driven malignancy
}

\begin{abstract}
Pavlos Missios, ${ }^{1,2,3}$ Edroaldo Lummertz da Rocha, ${ }^{1,2,3,4}$ Daniel S. Pearson,, ${ }^{1,2,3}$ Julia Philipp,,$^{5,6}$ Maria M. Aleman, ${ }^{7}$ Mehdi Pirouz, ${ }^{1,2,8}$ Dorian Farache, ${ }^{9,10}$ Joseph W. Franses, ${ }^{5}$ Caroline Kubaczka, ${ }^{1,2,3}$ Kaloyan M. Tsanov, ${ }^{1,2,3}$ Deepak K. Jha, ${ }^{1,2,3}$ Brian Pepe-Mooney, ${ }^{1,2}$ John T. Powers, ${ }^{1,2,3}$ Richard I. Gregory, ${ }^{1,2,3,11,12}$ Amy S.Y. Lee, ${ }^{9,10}$ Daniel Dominguez, ${ }^{7,13}$ David T. Ting, ${ }^{5}$ and George Q. Daley, ${ }^{1,2,3}$

${ }^{1}$ Stem Cell Program, Boston Children's Hospital, Boston, Massachusetts, USA. ²Division of Hematology/Oncology, Boston Children's Hospital and Dana Farber Cancer Institute, Boston, Massachusetts, USA. ${ }^{3}$ Department of Biological Chemistry and Molecular Pharmacology, Harvard Medical School, Boston, Massachusetts, USA. ${ }^{4}$ Department of Microbiology, Immunology and Parasitology, Federal University of Santa Catarina, Florianópolis, Brazil. ${ }^{5}$ Massachusetts General Hospital Cancer Center, Harvard Medical School, Boston, Massachusetts, USA. ${ }^{6}$ Department of Otorhinolaryngology, Head and Neck Surgery, University of Tübingen, Tübingen, Germany. 'Department of Pharmacology, University of North Carolina at Chapel Hill, Chapel Hill, North Carolina, USA. ${ }^{8}$ Center for Advanced Biotechnology and Medicine, Rutgers University, Piscataway, New Jersey, USA. ${ }^{9}$ Department of Cell Biology, Harvard Medical School, Boston, Massachusetts, USA. ${ }^{10}$ Department of Cancer Immunology and Virology, Dana-Farber Cancer Institute, Boston, Massachusetts, USA. ${ }^{11}$ Harvard Stem Cell Institute, Cambridge, Massachusetts, USA. ${ }^{2}$ Harvard Initiative for RNA Medicine, Boston, Massachusetts, USA. ${ }^{13}$ Lineberger Comprehensive Cancer Center, University of North Carolina at Chapel Hill, Chapel Hill, North Carolina, USA.
\end{abstract}

\begin{abstract}
High expression of LIN28B is associated with aggressive malignancy and poor survival. Here, probing MYCN-amplified neuroblastoma as a model system, we showed that LIN28B expression was associated with enhanced cell migration in vitro and invasive and metastatic behavior in murine xenografts. Sequence analysis of the polyribosome fraction of LIN28B-expressing neuroblastoma cells revealed let-7-independent enrichment of transcripts encoding components of the translational and ribosomal apparatus and depletion of transcripts of neuronal developmental programs. We further observed that LIN28B utilizes both its cold shock and zinc finger RNA binding domains to preferentially interact with MYCN-induced transcripts of the ribosomal complex, enhancing their translation. These data demonstrated that LIN28B couples the MYCN-driven transcriptional program to enhanced ribosomal translation, thereby implicating LIN28B as a posttranscriptional driver of the metastatic phenotype.
\end{abstract}

\section{Introduction}

Tight translational control of the transcriptome is a conserved regulatory mechanism during development and the physiological and pathological stress responses (1-3). Activation of ribosomal biogenesis plays a critical role in cancer initiation and progression (4), and perturbation of ribosomal genes inhibits MYC-induced tumorigenesis (5). In their role as rheostats of transcription, MYCN and other members of the MYC family induce global transcription of a broad range of mRNAs, including members of the protein-synthesis complex (6-10). Recently, dysregulation

Conflict of interest: GQD is a member of the scientific advisory board of, holds equity in, and receives consulting fees from 28/7 Therapeutics; GQD holds equity in Epizyme, Inc. and iTCells, Inc. DTT has received consulting fees from ROME Therapeutics, Foundation Medicine, Inc.; NanoString Technologies; EMD MilliporeSigma; and Pfizer. DTT is a founder and has equity in ROME Therapeutics, PanTher Therapeutics, and TellBio, Inc. DTT receives research support from ACD-Biotechne, PureTech Health LLC, and Ribon Therapeutics. DTT's interests were reviewed and are managed by Massachusetts General Hospital and Mass General Brigham in accordance with their conflict-of-interest policies. RIG is a member of the scientific advisory board and holds equity in and receives consulting fees from 28/7 Therapeutics and Theon Therapeutics. JWF has received consulting fees from Foundation Medicine, Inc. JWF receives research funding from Genentech.

Copyright: () 2021, American Society for Clinical Investigation.

Submitted: October 13, 2020; Accepted: September 21, 2021; Published: November 15, 2021.

Reference information: J Clin Invest. 2021;131(22):e145142.

https://doi.org/10.1172/JCl145142. of ribosomal protein expression and translation emerged as an enriched pathway in a screen for enhanced metastasis of breast cancer, indicating the importance of tight coupling of increased transcription and translation in cancer (11).

LIN28A and its paralog LIN28B are highly conserved RNA binding proteins and master regulators of developmental timing (12). LIN28 proteins are highly expressed during embryonic development and downregulated in most adult tissues. Their reactivation in a subset of cancers has established their function as oncofetal proteins (13-19). Multiple mechanisms for posttranscriptional gene regulation by LIN28 have been described, chiefly centered around its repression of the let-7 miRNA family $(20,21)$. As a well-established tumor suppressor miRNA, let-7 negatively regulates master regulators of stemness and oncogenes like MYC, RAS, Hmga2, and others (22-24). LIN28 also binds and regulates a large set of mRNAs, independent of let-7 (25-28). Recently, LIN28B was shown to drive transcriptome-wide changes in the translational program, implicating a regulatory function of LIN28B in translation (29).

Previously, LIN28 protein expression has been reported to coincide with expression of MYC-family members in different tumors (30-33). MYCN and MYC have been shown to induce LIN28B expression by binding to its promoter region $(30,31,34)$. Furthermore, LIN28 proteins suppress processing of let-7 miRNA, which results in higher expression of $M Y C / M Y C N$, whose 3 '-UTRs harbor let-7 binding sites $(31,34)$. The coordinated regulation of the LIN28 and MYC/MYCN oncoproteins has been 
interrogated at the mRNA level, but less attention has been paid to effects on translation.

Here, we showed that LIN28B acts as a rheostat of translation via direct posttranscriptional alteration of ribosomal dynamics in $M Y C N$-amplified neuroblastoma, a prototypic model for MYCN-driven tumorigenesis, resulting in a dedifferentiated and metastatic cell state. Our results position the LIN28B protein as an important coregulator with MYCN in tumor progression, coupling enhanced translation with the enhanced transcriptional program.

\section{Results}

LIN28B mediates features of aggressive disease in a MYCN-driven tumor model. Despite the identification of LIN28B as an oncoprotein, genetic loss of $L I N 28 B$ has a negligible effect on $2 \mathrm{D}$ cell growth and proliferation in MYCN-driven neuroblastoma cell lines in vitro (ref. 34 and Supplemental Figure 1, A-C; supplemental material available online with this article; https://doi. org/10.1172/JCI145142DS1). Thus, we sought to investigate the in vivo behavior of neuroblastoma with regard to LIN28B expression. First, we assessed the survival of immunocompromised mice after tail vein injection of various neuroblastoma cell lines. Mice injected with the LIN28B-expressing neuroblastoma cell lines BE2C and Kelly showed reduced survival relative to mice injected with non-LIN28B-expressing cell lines SK-N-AS, SY5Y, and CHP-212 (Supplemental Figure 1, D and E). Moreover, CRISPR-mediated genetic deletion of LIN28B in BE2C cells led to a markedly reduced bioluminescent signal 4 weeks after injection, indicating reduced tumor mass in livers of injected mice (Figure 1, A and B), and loss of LIN28B in BE2C and Kelly cells led to prolonged lifespan in injected mice (Figure 1, C and D). A limiting-dilution assay via tail vein injection demonstrated that $L I N 28 B$ deficiency led to a markedly reduced frequency of tumor-initiating cells (Figure $1 \mathrm{E}$ ) and profoundly reduced tumor burden (Supplemental Figure 1, F and G).

Whereas altered cell proliferation is a common feature of cancer, metastatic potential has been linked with abnormalities of cellular migration and invasiveness. Consistent with the metastatic phenotype of enhanced liver colonization in vivo, LIN28Bexpressing cells showed increased anchorage-independent growth, cell migration, and cell invasion in vitro (Supplemental Figure 2).

Taken together, these results indicate that LIN28B promotes pathological features of aggressive malignancy in vivo and cellular phenotypes associated with increased migratory and invasive behaviors in vitro.

LIN28B mediates metastasis of MYCN-amplified neuroblastoma cells. To directly assess metastatic potential in an orthogonal assay, we formed primary tumors by injection of $M Y C N$-amplified neuroblastoma cells under the kidney capsule followed by bioluminescence tracking (Figure 2A). Consistent with our results from tail vein injection assays, we detected liver metastases when LIN28B-expressing BE2C neuroblastoma cells were injected under the left kidney capsule (Figure 2B), and we observed greater metastatic burden in the livers of mice injected with BE2C CTRL neuroblastoma cells relative to LIN28B-deficient cells (Figure 2, B-D, and Supplemental Figure 3, A-D). Similarly, Kelly CTRL cells metastasized significantly more than the LIN28B-KO counterparts (Figure 2, E and F). Of note, primary tumors from Kelly cells also exhibited reduced bioluminescent signal after loss of $L I N 28 B$ (Supplemental Figure 3, E and F). In contrast to BE2C, Kelly LIN28B-KO tumors harvested from the primary site of injection showed LIN28B protein expression, suggesting that tumor growth resulted from cells that had escaped the LIN28B population KO (Supplemental Figure $3, \mathrm{G}$ and $\mathrm{H}$ ). Direct comparison of liver and primary tumor bioluminescence revealed higher metastatic burden in LIN28B-expressing $\mathrm{BE} 2 \mathrm{C}$ and Kelly cells, independent of the primary growth deficit in Kelly cells (Supplemental Figure 3, I and J).

To evaluate the therapeutic potential of targeting $L I N 28 B$ in patients with MYCN-induced neuroblastoma, we obtained luciferase-expressing stage $4 \mathrm{MYCN}$-amplified neuroblastoma patient-derived xenograft (PDX) models that had been shown to metastasize into livers of immunocompromised mice (35). To assess the effect of LIN28B depletion on the PDX line with the highest LIN28B mRNA expression, we delivered highly concentrated CRISPR/Cas 9-lentivirus targeting LIN28B, followed by orthotopic reimplantation in the left adrenal gland (Supplemental Figure 4, A and B). We maintained a small fraction of infected cells in neurosphere media and confirmed high-efficiency transduction as measured by strong mCherry expression (Supplemental Figure $4 \mathrm{C}$ ). Further, quantitative PCR (qPCR) analysis showed decreased transcript expression of $L I N 28 B$ and de-repression of let-7 miRNA (Supplemental Figure 4D). Four weeks after reimplantation of the genetically modified PDX lines, we assessed metastatic spread to the liver using bioluminescence imaging. Again, LIN28B depletion mediated a substantially lower metastatic burden (Figure 2, G and $\mathrm{H}$, and Supplemental Figure 4, E-G).

LIN28B RNA binding function is required to promote aggressive disease. To evaluate the role of RNA binding, we reexpressed the normal WT LIN28B coding sequence (KO+WT) or an RNA binding mutant of LIN28B (KO+MUT) after transient CRISPR/Cas 9-mediated KO (Supplemental Figure 5). We then assayed the relative behavior of these cells by dual color competition, comparing GFP-labeled LIN28B protein-expressing cells with RFP-labeled KO cells (Figure $3 \mathrm{~A})$. We injected both genotypes in equal numbers into the tail vein of immunocompromised mice. Mice injected with cells expressing intact WT LIN28B exhibited higher tumor burden at 5 weeks compared with those injected with cells expressing the RNA binding mutant (MUT) (Figure 3B). Furthermore, cells with LIN28B WT protein expression showed significantly higher relative contribution to malignant ascites of injected mice, indicating far greater metastatic potential than the cells carrying LIN28B MUT (Figure 3C).

Finally, we modified our kidney capsule injection protocol to include nephrectomy 4 days after tumor cell injection, which prolonged lifespan and enabled investigation of relative metastatic behavior by direct cell competition (Figure 3A). Again, in vivo bioluminescence imaging showed a greater metastatic burden after LIN28B reexpression, whereas a metastatic phenotype was not observed when a LIN28B mutant construct lacking RNA binding capacity was reexpressed (Figure 3D). In flow cytometry analysis of single-cell dissociated livers, LIN28B WT-expressing cells showed significantly higher contribution to metastases relative to the $\mathrm{KO}$ genotype (Figure 3E); again, the mutant protein failed to demonstrate a metastatic phenotype.

To further demonstrate a link between LIN28B expression and metastatic phenotypes in neuroblastoma cells, we showed 
A

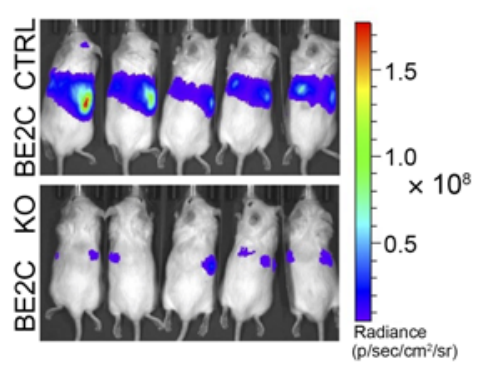

D

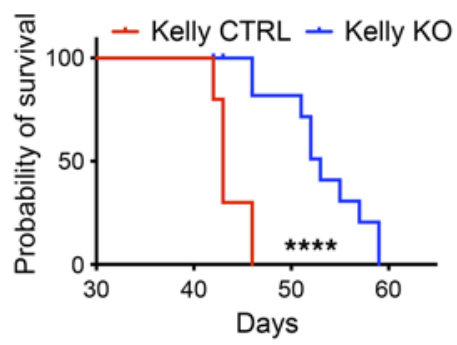

B
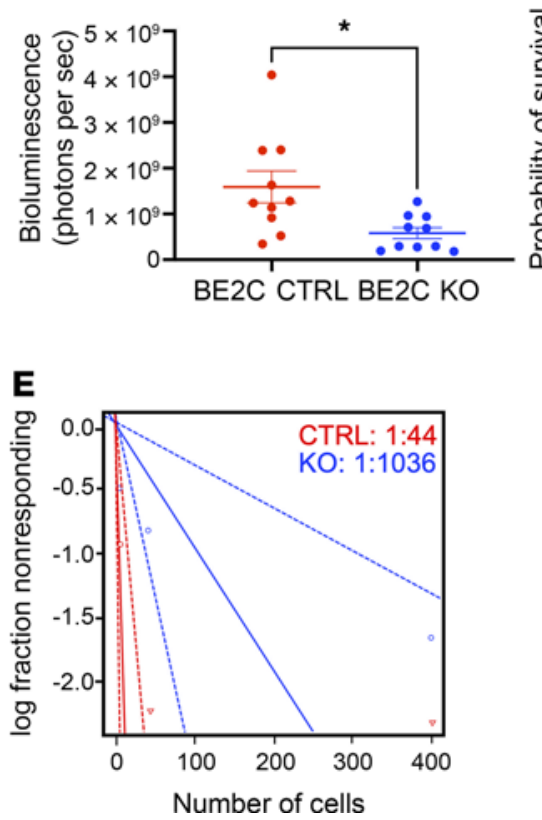

C

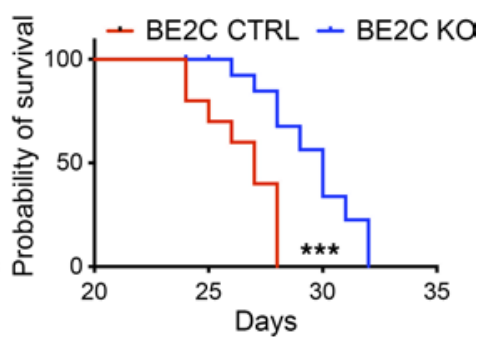

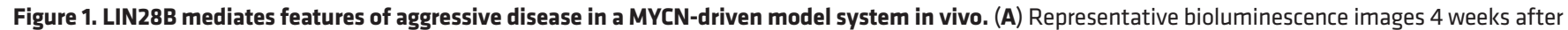
injection of luciferase-expressing BE2C cells of the indicated genotypes into the tail vein of immunocompromised mice (NSC). $n=5$ for BE2C control (CTRL1 gRNA) and $n=5$ for BE2C KO (EX3.1 gRNA). (B) Quantitative analysis of bioluminescence of mice used in A. $n=5 /$ specific gRNA (CTRL1/2 for CTRL; EX2 and EX3.1 for KO), $n=10$ /group. (C) Kaplan-Meier survival curve of NSC mice injected with 100,000 BE2C cells of the indicated genotypes. $n=5 /$ specific gRNA (CTRL1/2 for CTRL; EX2 and EX3.1 for KO), $n=10$ /group. (D) Kaplan-Meier survival curve of NSG mice injected with 100,000 Kelly cells of the indicated genotypes. $n=5$ /specific gRNA (CTRL1/2 for CTRL; EX2 and EX3.1 for KO), $n=10 /$ group. (E) Limiting-dilution tail vein injection assay of BE2C cells infected with a modified V2 CRISPR/Cas9 lentivirus expressing mCherry (CTRL1 [CTRL] vs. EX3.1 [KO] gRNA; $n=5 /$ mice per dilution). All statistical data were assessed using log-rank (Mantel-Cox) test (C and D) and 2-tailed Student's $t$ test (B) and are presented as mean \pm SEM. ${ }^{*} P<0.05 ;{ }^{* *} P<0.001 ;{ }^{* * *} P<0.0001$.

that enforced expression of LIN28B WT but not LIN28B MUT protein in non-LIN28B-expressing neuroblastoma cell lines CHP-212, SK-N-AS, and SY5Y led to enhanced migration in vitro (Supplemental Figure 6).

Taken together, these data underscore the migratory and metastatic potential mediated by LIN28B and indicate that the metastatic phenotype is dependent on its RNA binding activity.

LIN28B alters polysome enrichment and defines a prometastatic transcriptional cell state. To obtain mechanistic insights regarding the role of LIN28B in facilitating metastatic disease, we performed total mRNA-Seq as well as mRNA-Seq of the polyribosome (polysome) fraction in BE2C CTRL and LIN28B-KO cells (Figure 4A). Loss of LIN28B had a larger impact on polysome-associated transcripts than on bulk mRNA expression (Figure 4B), confirming a role for LIN28B in the regulation of translation, as reported (29). Gene set enrichment analyses (GSEA) of bulk mRNA expression revealed strong enrichment for gene sets of the epithelial-mesenchymal transition (EMT) pathway and metastasis (Figure 4C). In contrast, GO term analyses of differentially expressed genes after loss of LIN28B revealed a strong neurogenesis signature, resembling induction of developmental programs of the neural crest (Supplemental Figure 7A).

Despite a strong repression of let-7 miRNA coincident with LIN28B expression (Supplemental Figure 1B), let-7 targets did not exhibit higher polysome enrichment in BE2C CTRL cells (Figure 4D).

Gene ontology (GO) analyses on differentially polysomeenriched transcripts revealed a strong induction of translation and ribosome-related pathways in LIN28B-expressing cells (Figure 5A). Indeed, many transcripts encoding for ribosomal proteins were enriched in the polysome fraction under LIN28B expression (Figure $5 \mathrm{~B})$. In contrast, loss of LIN28B led to strong enrichment of transcripts characteristic of neuronal differentiation programs (Figure $5 \mathrm{~A}$ ).

Analysis of transcript properties in LIN28B-expressing BE2C cells revealed significant polysome enrichment for transcripts with higher adenine/uridine (AU) content within the 3'-UTR and coding sequence (Figure 5C). Furthermore, transcripts within gene sets whose GO terms related to cell/neuronal development as well as RNA processing and translation indicated a trend toward lower AU content for developmental programs of the neural lineage (Figure 5D.)

To uncover the protein binding partners of LIN28B in BE2C cells, we performed IP followed by mass spectrometry. Interestingly, the majority of LIN28B protein binding partners were classified as RNA binding proteins themselves (Supplemental Figure 7B). For those with defined, published binding motifs, we detected several cases of AU-rich binding proteins (Supplemental Figure 7C).

Together, these data revealed that LIN28B induced polysome enrichment of AU-rich transcripts encoding proteins of the ribosomal apparatus.

LIN28B cooperates with MYCN to alter ribosomal dynamics. To identify the distinct mRNA binding targets of LIN28B in BE2C neuroblastoma cells, we performed RNA IP coupled with polyA-selected RNA-Seq (RIP-Seq) using either FLAG-tagged WT or an RNA binding-deficient LIN28B protein (Supplemental 
A

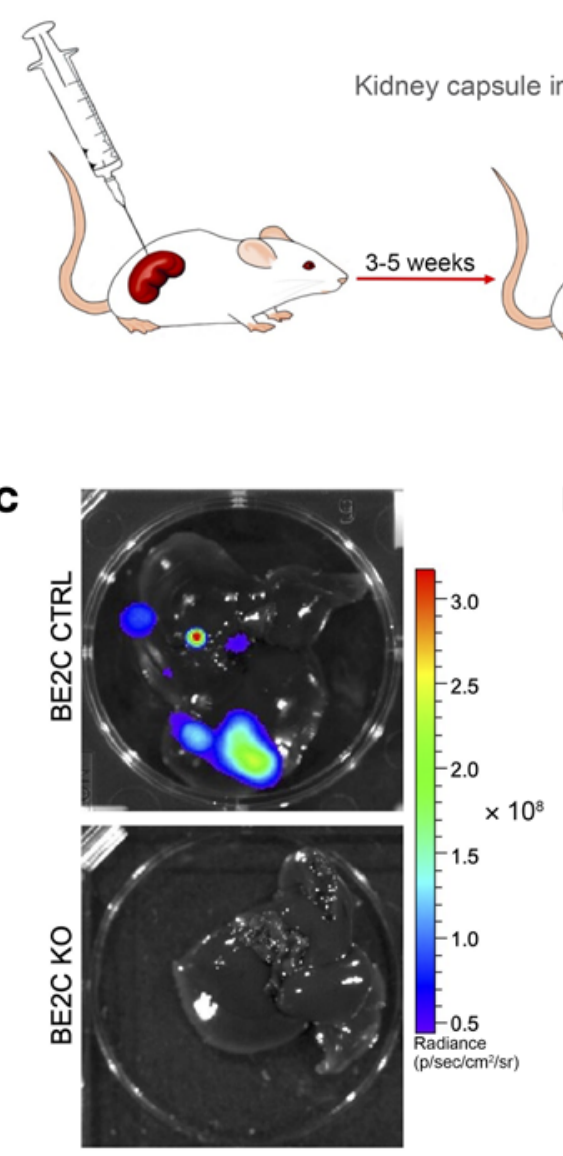

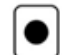

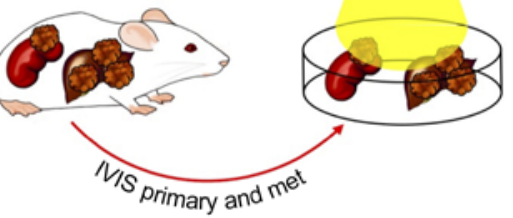

$\mathbf{E}$

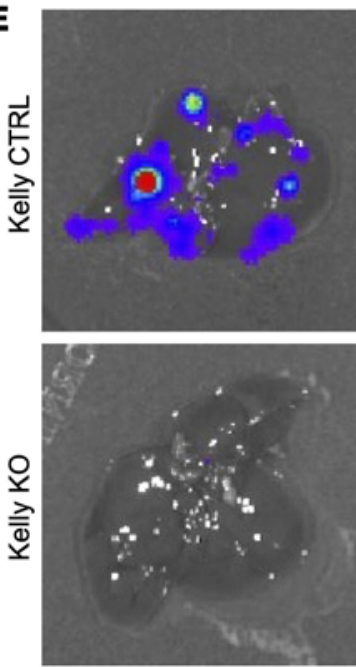

B

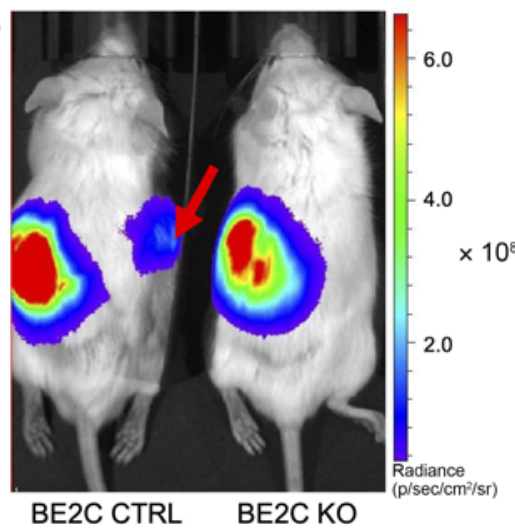

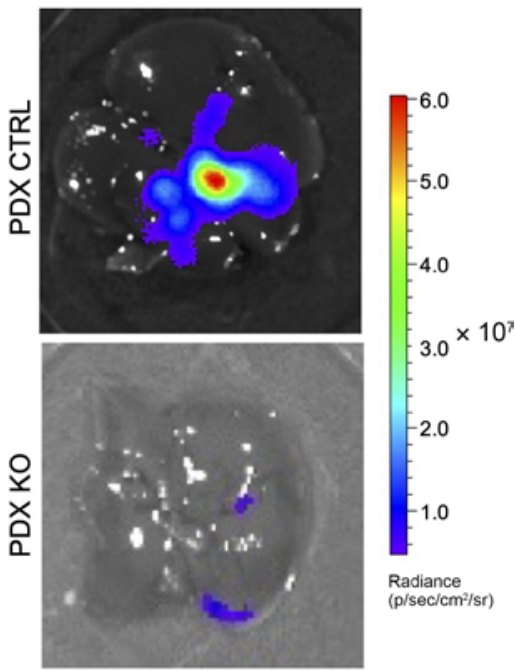

D

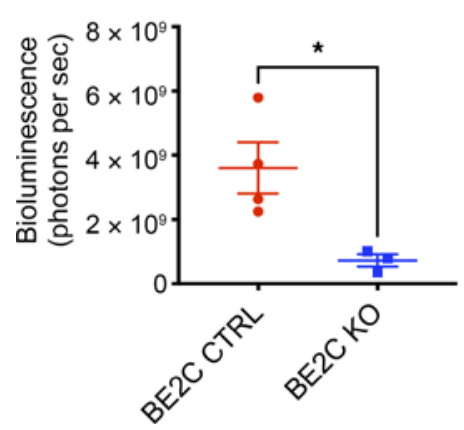

$\mathbf{F}$

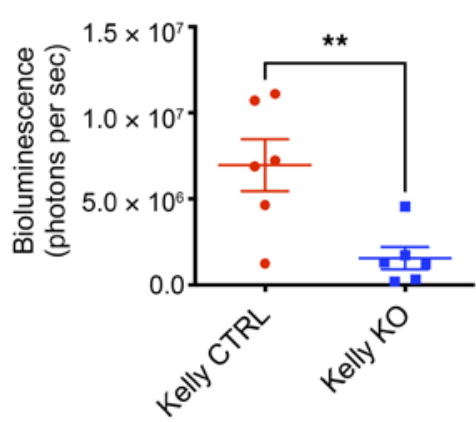

H

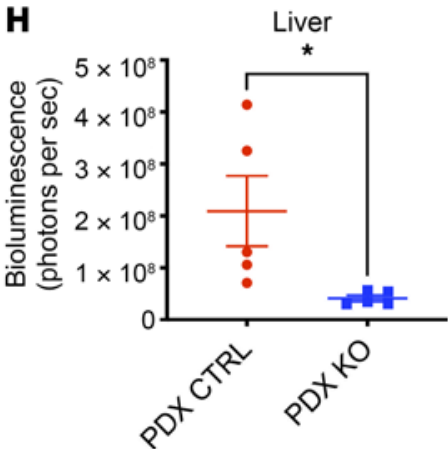

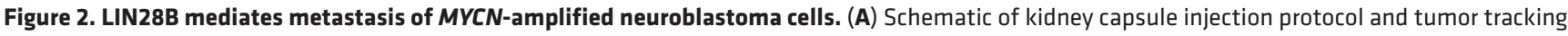
(primary and metastatic [Met]) using an in vivo imaging system (IVIS). (B) Representative bioluminescence image of mice 3 weeks after injection of BE2C cells (CTRL) or BE2C cells lacking LIN28B expression (KO) under the capsule of the left kidney. Red arrow pointing to metastatic site. (C) Representative bioluminescence images of explanted livers 3 weeks after injection of BE2C cells under the kidney capsule of NSC mice. (D) Quantitative analysis of bioluminescence of livers of indicated groups ( $n=4$ mice for BE2C CTRL and $n=3$ mice for BE2C-KO cells). (E) Representative bioluminescence images of explanted livers 5 weeks after injection of Kelly cells under the kidney capsule of NSG mice. (F) Quantitative analysis of bioluminescence of livers of indicated groups ( $n=6$ mice/group). (G) Representative bioluminescence image of explanted livers 4 weeks after orthotopic injection of LIN28B-expressing patient derived xenograft (PDX) cells. (H) Quantitative analysis of bioluminescence of livers of indicated groups ( $n=5$ mice/group). All statistical data were assessed using 2-tailed Student's $t$ test (D, F, and $\mathbf{H}$ ) and are presented as mean \pm SEM. ${ }^{*} P<0.05 ;{ }^{* *} P<0.01$.

Figure 5D). Interestingly, LIN28B-bound transcripts exhibited high AU content in their coding and 3'-UTR region (Supplemental Figure 7D). Comparing the RIP-Seq and RNA-Seq data sets for BE2C cells, we observed a significant depletion of bound mRNA transcripts after loss of LIN28B, directly implicating LIN28B in this context (Supplemental Figure 7E). Differential expression of
LIN28B bound transcripts was independent of their status as let-7 targets (Supplemental Figure 7F).

We detected a significant overlap of LIN28B-bound mRNA targets and transcripts with differential polysome enrichment in BE2C CTRL cells. Indeed, the majority of overlapped targets exhibited increased polysome enrichment (Figure 6A). Further analyses 
A

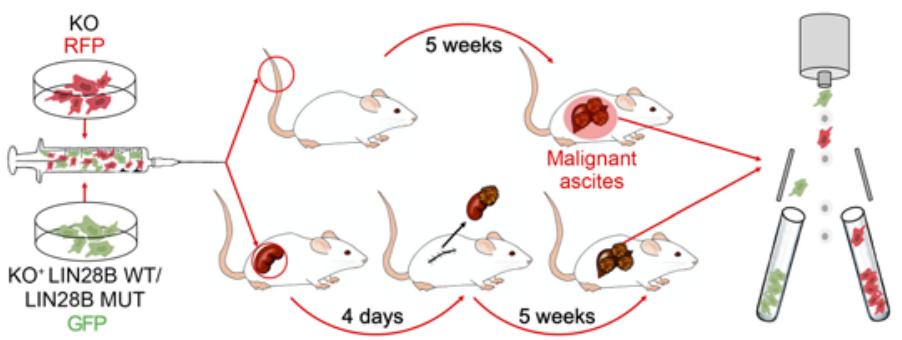

B
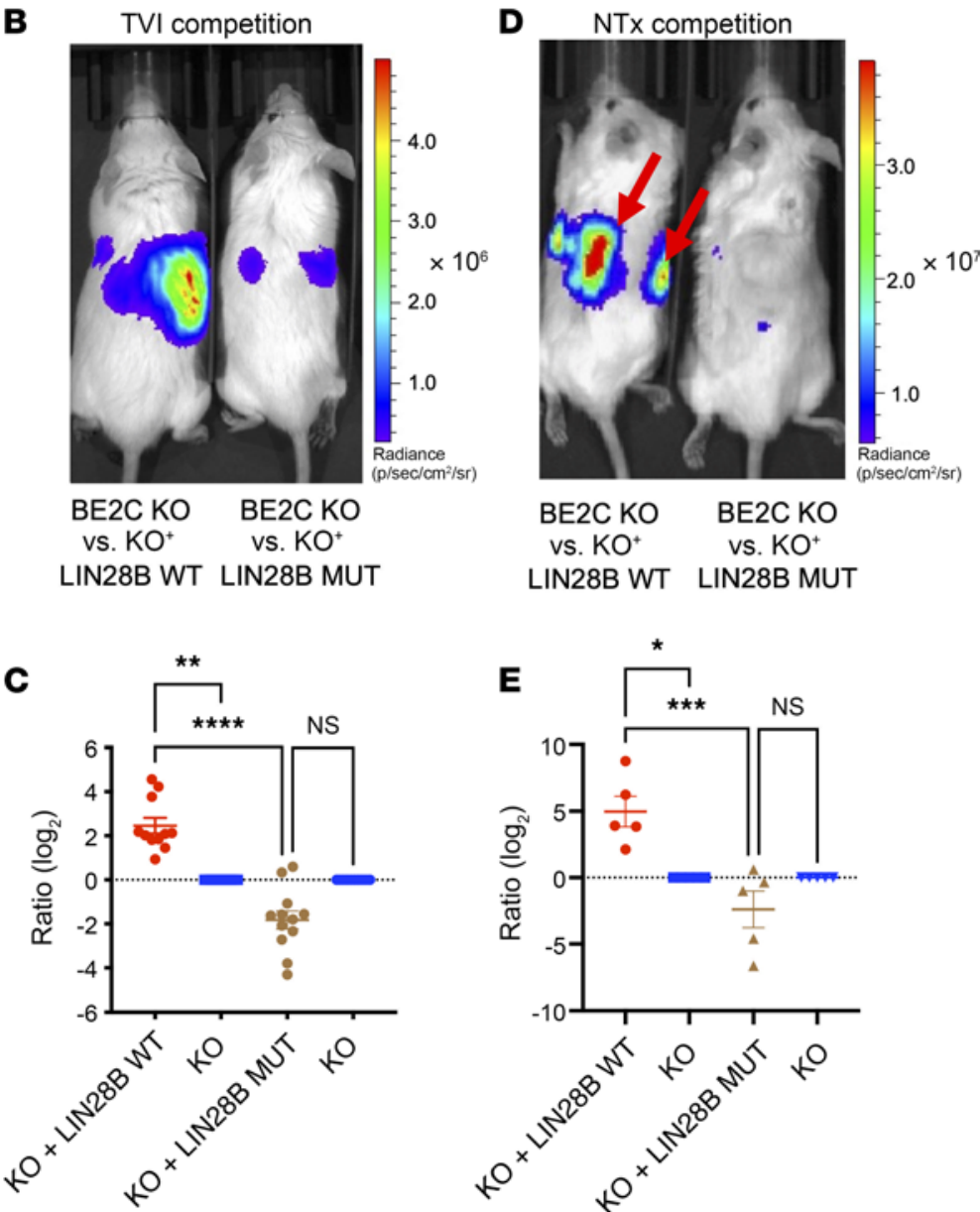

Figure 3. LIN28B RNA binding function is required to promote aggressive disease. (A) Schematic of the dual-color competition experiment: mice were injected with a mixture of equal parts of LIN28B-KO cells labeled with RFP and LIN28B-KO cells carrying a GFP marker and reexpressing WT LIN28B (LIN28B WT) or an RNA binding mutant of LIN28B (LIN28B MUT). Upper row: mice were injected by tail vein with both cell populations followed by analysis of the malignant ascites via flow cytometry (C and B). Lower row: mice were injected with cells under the kidney capsule followed by nephrectomy (NTx) 4 days later; single-cell suspensions were then prepared from livers. Final analysis was performed by flow cytometry (D and E). (B) Representative bioluminescence image of NSG mice 5 weeks after tail vein injection of the indicated mixtures. (C) Graph depicts the ratio of GFP-labeled cells (KO+LIN28B WT/MUT) to RFP-labeled cells (KO) isolated from ascites and measured by flow cytometry. KO cells were set to 1 ( $n=11$ mice for the KO/KO+WT and $n=$ 12 mice for the KO/KO+MUT competition). (D) Representative bioluminescence image of mice with liver metastases 5 weeks after resection of a primary tumor that resulted from injection of a mixture of BE2C KO (RFP) and BE2C KO+LIN28B WT or BE2C KO+LIN28B MUT (GFP) under the kidney capsule. Red arrows pointing to metastatic sites. (E) Graph depicts the ratio of GFP-positive cells (KO+LIN28B WT/MUT) to RFP-positive cells (KO) isolated from livers harboring metastatic tumors for mice injected with the indicated cells. KO cells were set to 1 ( $n$ $=5$ mice $/$ group). All statistical data were assessed using the Kruskal-Wallis test ( $\mathbf{C}$ and $\mathbf{E}$ ) and are presented as mean \pm SEM. ${ }^{*} P<0.05{ }^{* *} P<0.01{ }^{* * *} P<0.001{ }^{* * * *} P<0.0001$.

programs and members of the ribosome, similar to our MYCN model system (Supplemental Figure 8, B and C, and refs. 26, 27, 36). When comparing polysome-enriched targets under conditions of variable LIN28B expression in HEK293T cells, we observed a trend toward positive polysome enrichment of LIN28B RIP-Seq targets with increasing LIN28B protein expression (Supplemental Figure 8D).

The mRNA for the ribosomal protein RPS29, which is part of the small ribosomal subunit and a MYCN target, showed significant binding by LIN28B and polysome enrichment (Figure 6C). We took advantage of an in vitro translation system that allows separation of the contribution of translation regulation from transcription and miRNA targeting to test for the influence of the different LIN28B proteins on RPS29 translation. Native LIN28B protein of those LIN28B-bound transcripts that also showed polysome enrichment revealed significant overlap with MYC CHIP-Seq targets (Supplemental Figure 8A and ref. 36) as well as GO pathways of ribosomal biogenesis and translation, suggesting increased translation of these transcripts (Figure 6B). Indeed, LIN28B showed significant binding to MYCN-induced transcripts in BE2C neuroblastoma cells (Figure 6A), and GO analyses revealed enrichment for translational programs and components of the ribosome (Figure $6, \mathrm{~B}$ and C). Additionally, we observed that global protein synthesis was increased in LIN28B-expressing BE2C cells, as determined by metabolic labeling with S35 methionine (Figure 6D).

We then compared publicly available MYC CHIP-Seq, LIN28B CLIP-Seq, and polysome sequencing with our RIP-Seq data sets. LIN28B CLIP-Seq and MYC CHIP-Seq targets in HEK293T cells showed significant overlap and enrichment for translational
(WT) induced translation of the RPS29 reporter compared with the LIN28 RNA binding mutant in extracts made from BE2C and other $M Y C N$-expressing cell lines (Figure 6E and Supplemental Figure 8E).

To evaluate domain-specific binding, we overexpressed LIN28B RNA binding-deficient mutants in either the zinc knuckle domain (ZKD) or the cold shock domain (CSD). Interestingly, both single mutants shared the vast majority of binding partners, indicating mutual binding of both domains to the same transcripts (Supplemental Figure 8F). Again, GO term analysis of overlapping targets revealed members of the translation machinery (Supplemental Figure 9A). Furthermore, binding partners specific to the CSD showed preferential polysome enrichment (Supplemental Figure $8 \mathrm{~F}$ ), whereas binding partners specific to the ZKD were depleted in LIN28B-expressing BE2C cells (Supplemental Figure $8 \mathrm{~F})$. We detected a preferential binding of the CSD to AU-rich 
A
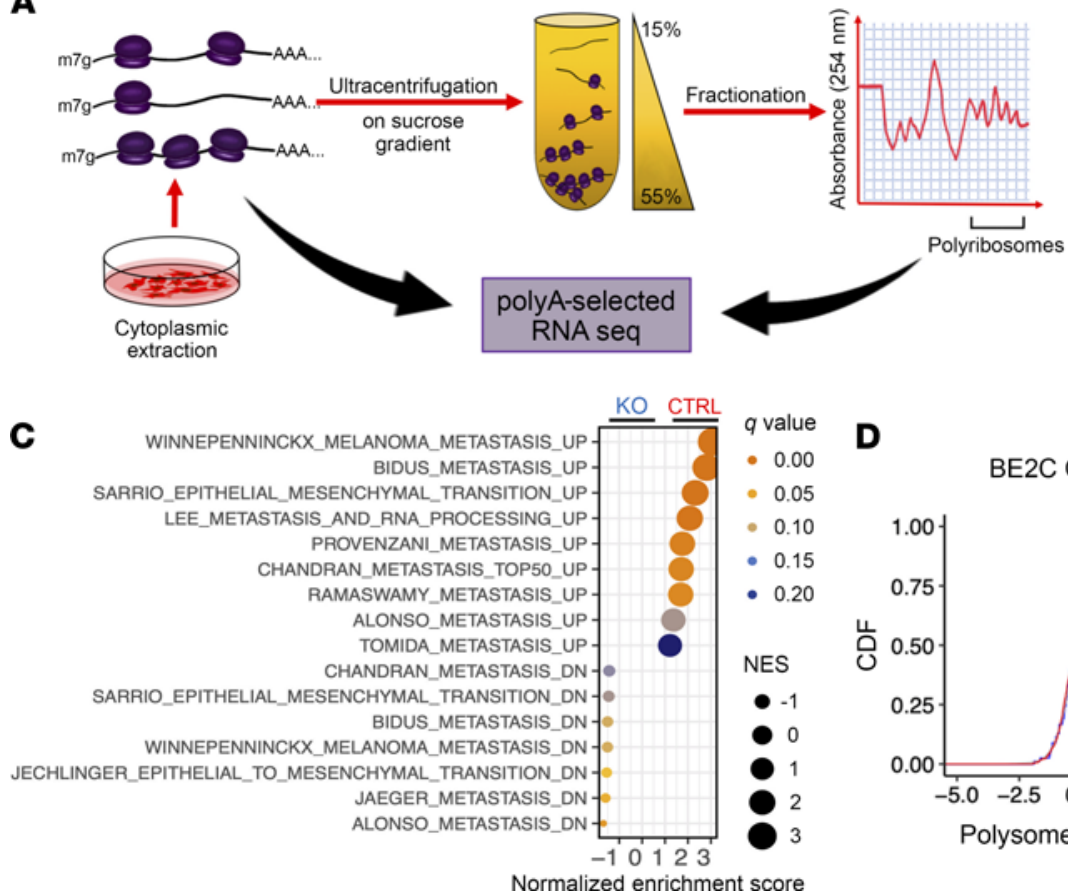
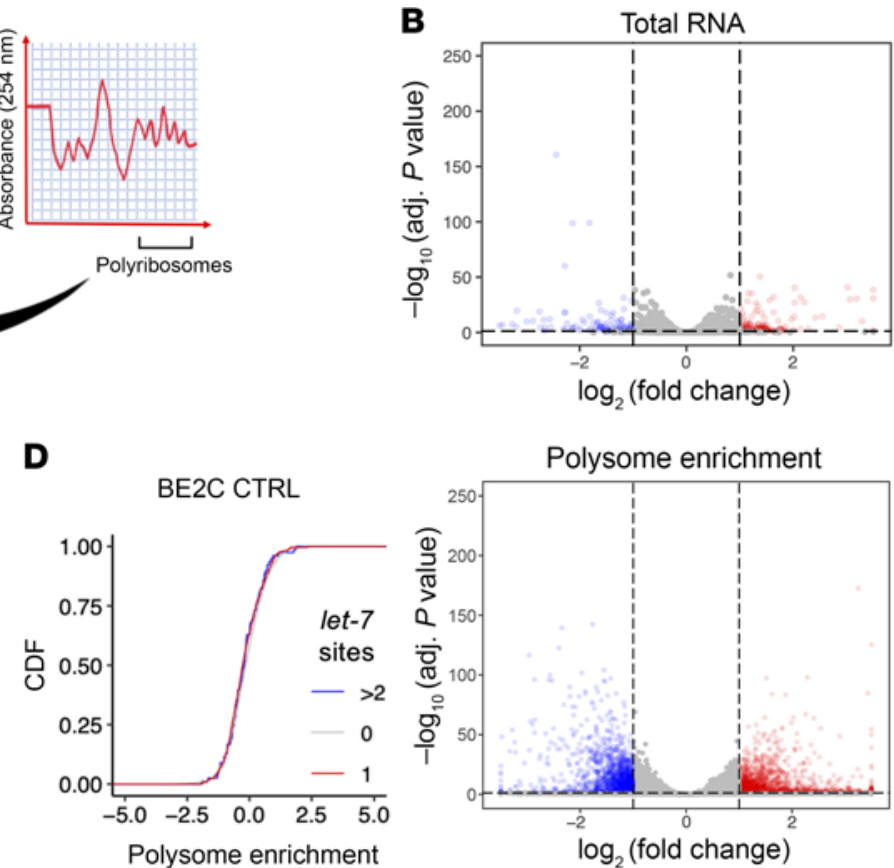

Figure 4. LIN28B alters polysome enrichment and defines a prometastatic transcriptional cell state. (A) Overview of the experimental design for extraction of total RNA and RNA of the polyribosome (polysome) fraction followed by polyA-selected RNA-Seq. (B) Volcano plots representing the differentially expressed transcripts in the whole transcriptome (total RNA) and transcripts enriched in the polysome fraction (polysome enrichment) in BE2C CTRL compared with BE2C LIN28B-KO cells. (C) Gene set enrichment analysis (CSEA) of differentially expressed genes in BE2C. (D) Cumulative distribution function (CDF) of differentially polysome-enriched transcripts in BE2C CTRL harboring more than 2 (blue), 1 (red), or 0 (gray) conserved let-7 binding sites. Differential comparisons were made between BE2C CTRL and BE2C LIN28B-KO cells.

transcripts, whereas ZKD bound to transcripts with lower $\mathrm{AU}$ content (Supplemental Figure 9B), supporting the reported binding motif for the ZKD (21). Reexpression of either single mutant LIN28B protein alone was insufficient to phenocopy the migratory phenotype associated with LIN28B WT protein in vitro (Supplemental Figure 9, C and D, and Supplemental Figure 10, A-F). These results suggest that, similar to the inhibition of let-7 (21), binding by both RNA binding sites appears to be required for proper posttranscriptional regulation of bound targets.

Taken together, our data revealed that LIN28B binds to and accounts for polyribosome enrichment of MYCN- and MYCdriven mRNA transcripts of the ribosomal apparatus.

LIN28B expression correlates with MYCN and ribosomal biogenesis. LIN28B expression has been associated with advanced-stage disease in multiple tumor entities $(13,15-17)$. To corroborate our observations for neuroblastoma and interrogate molecular mechanisms accounting for disease state, we reanalyzed 2 of the most extensive cohort studies comprising data from over 1000 neuroblastoma patients (37, 38). In line with previously published data sets for neuroblastoma, high expression of LIN28B was associated with poor survival (Figure 7A and Supplemental Figure 11A). Furthermore, LIN28B expression correlated with later tumor stage in both studies (Supplemental Figure 11, B and C). To test whether LIN28B had a significant effect in the presence of MYCN amplification, we assessed survival in relation to $L I N 28 B$ expression in the $M Y C N$-amplified cohort. Interestingly, LIN28B expression correlated with worse survival even in the presence of MYCN amplification (Supplemental Figure 11D).
Next, we identified stage-specific signature genes from both data sets. The gene sets for stage 4 tumors of both independent studies showed considerable overlap (Supplemental Figure 11E). In line with our sequencing results, these common genes of the stage 4 signature revealed high enrichment for transcripts encoding for components of the ribosome (Supplemental Figure 11F). We then used these signature genes to train random forest transcriptional classifiers for each tumor stage. First, we tested these algorithms on our MYCN-driven model system. The LIN28B-expressing neuroblastoma line $\mathrm{BE} 2 \mathrm{C}$ was classified as a stage 4 neuroblastoma tumor, whereas loss of $L I N 28 B$ led not only to a complete reversal of the stage 4 signature, but reappearance of stage 1 signature genes in both independent studies, indicating significant loss of metastatic potential (Figure 7B and Supplemental Figure 11G).

Similar to LIN28B, RPS29 expression correlated with worse survival (Supplemental Figure 12A). siRNA-mediated knockdown of RPS29 led to impaired migratory potential in both $M Y C N$-amplified cell lines, BE2C and Kelly (Figure 7C and Supplemental Figure 12, B-E).

Treatment with retinoic acid after completion of intensive chemoradiotherapy reduces recurrence of late-stage neuroblastoma $(39,40)$. Genetic deletion of LIN28B sensitized BE2C neuroblastoma cells to retinoic acid, leading to enhanced growth arrest, reduced colony-forming capacity, and morphological differentiation in vitro (Figure 7, D-F, Supplemental Figure 13, A-C, and Supplemental Videos 1 and 2).

Coexpression analyses revealed a statistically significant correlation between LIN28B and MYCN not only in the above analyzed 
A

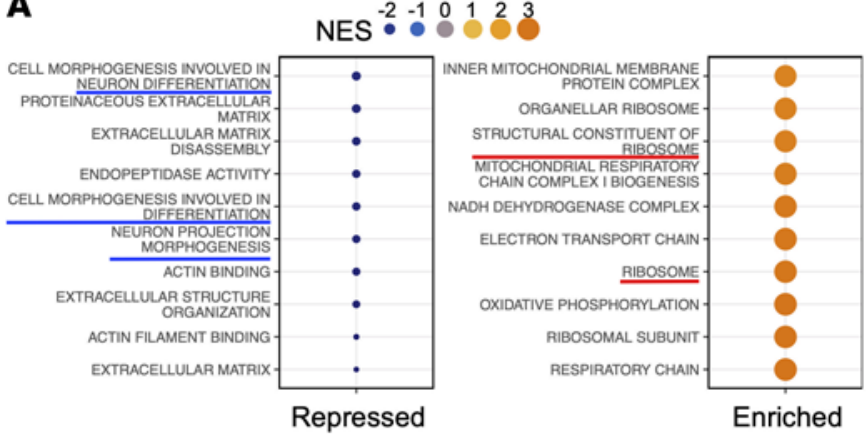

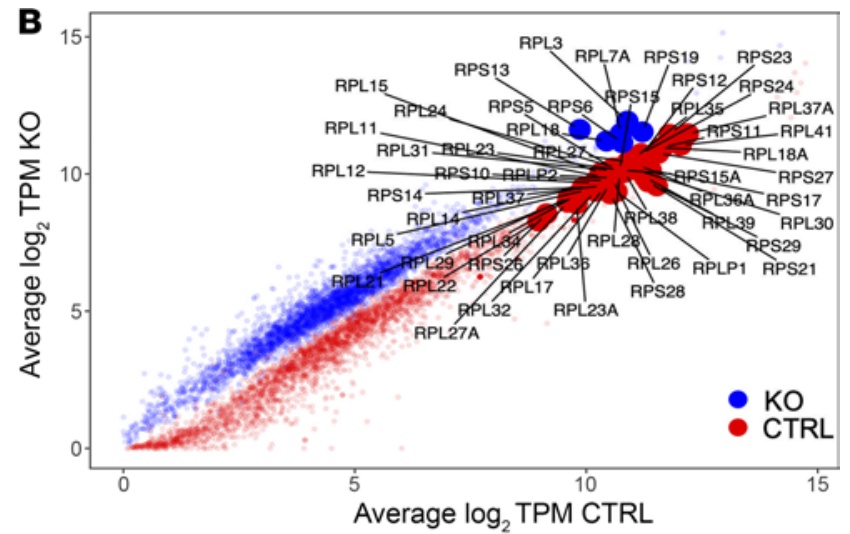

D

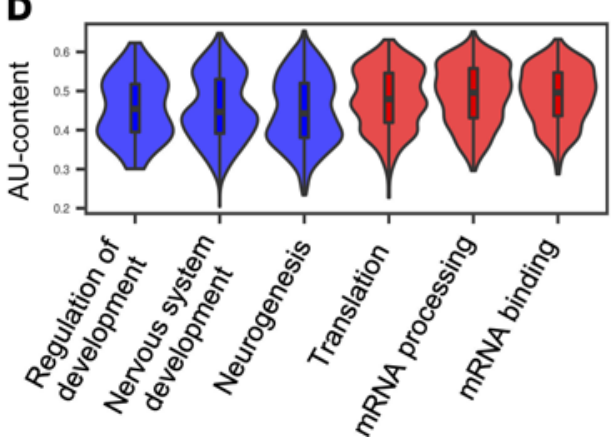

Figure 5. LIN28B induces polysome enrichment of AU-rich transcripts of the ribosomal apparatus. (A) Gene set enrichment analysis (GSEA) of differentially polysome-enriched transcripts in LIN28B-expressing BE2C cells. (B) Scatterplot depicts significant changes of differentially expressed transcripts of the polysome fraction. Ribosomal protein genes are indicated. (C) Violin plot depicting adenine-uracil (AU) content of differentially polysome-enriched transcripts separated for 5'-UTR, coding sequence (Coding), and 3'-UTR in BE2C CTRL. (D) The violin plot depicts AU content of coding sequence of transcripts belonging to the indicated GO gene sets. Differential comparisons were made between BE2C CTRL and BE2C LIN28B-KO cells.

cohort studies (Supplemental Figure 13, D and E) but throughout 31 annotated data sets from The Cancer Genome Atlas (TCGA) comprising data from over 9000 patients (Figure 7G). Finally, we queried genes positively correlated with $L I N 28 B$ in several publicly available data sets of $M Y C N$-expressing tumor types. Strikingly, LIN28B significantly correlated with transcripts belonging to the ribosome or other components of the protein-synthesis complex (Figure $7 \mathrm{H}$ ). These results suggest a coordinated action of $L I N 28 B$ and MYCN in multiple cancer types.

\section{Discussion}

Our work has revealed the prometastatic effect of LIN28B in $M Y C N$-amplified neuroblastoma cell lines and PDX samples, providing a compelling explanation for the association of high LIN28B expression with advanced cancer and poor prognosis. While $L I N 28 B$ deficiency has paradoxically shown rather weak to nonexistent proliferative effects in 2D growth assays for $M Y C N$ - and $M Y C$-expressing cancers in vitro $(34,41)$, here we have demonstrated that genetic loss of LIN28B led to loss of metastatic potential, reflected by reduced migratory and invasive phenotypes of MYCN-driven neuroblastoma in vitro as well as markedly reduced metastatic behavior in vivo. LIN28B KO had a prodifferentiation effect on $\mathrm{BE} 2 \mathrm{C}$ cells, as reflected by transcriptional induction of developmental programs of the neural crest. This phenomenon was amplified by cotreatment with retinoic acid, an agent with differentiation-inducing effects that has clinical utility in neuroblastoma and other MYC- and MYCNexpressing cancers (42).

Our experiments point toward a mechanism whereby LIN28B binds to MYCN-induced transcripts of the ribosomal apparatus to promote their polysome enrichment and translation. Our analyses shed light on the relationship between MYCN and LIN28B and highlight the importance of coordinated gene expression regulation by transcriptional and translational rheostats in cancer. The different members of the MYC family exhibit high structural homology and show functional redundancy $(43,44)$. Thus, our observations regarding LIN28B in MYCN-driven neuroblastoma could have important implications for MYC-driven tumors as well. Indeed, LIN28B showed significant binding to MYC-induced ribosomal transcripts in HEK293T cells (Supplemental Figure 7). Furthermore, loss of Lin28 had only a marginal effect on cell proliferation in Myc-driven liver cancer but correlated with tumorigenic potential in murine models (14), similar to our observations in the MYCN-driven tumor model of neuroblastoma.

Translational control has been implicated as a key phenomenon during tumorigenesis and tumor maintenance (45-49). Recently, dysregulation of translation has emerged as an important mechanism in late-stage, metastatic disease. Ribosomal biogenesis has been shown to be a driver of the EMT pathway (50). Furthermore, high ribosomal content and increased translation have been defined as hallmarks of highly metastatic circulating tumor cells in breast cancer (11). Increased expression of 
A

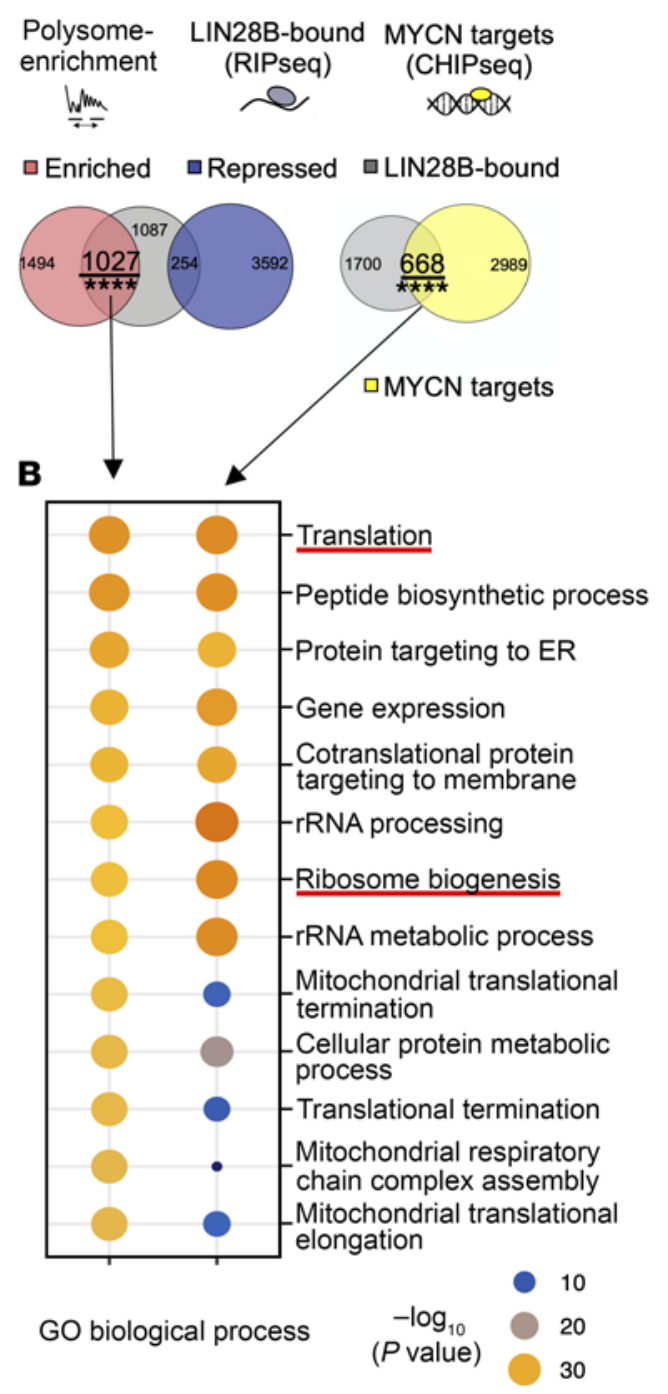

C

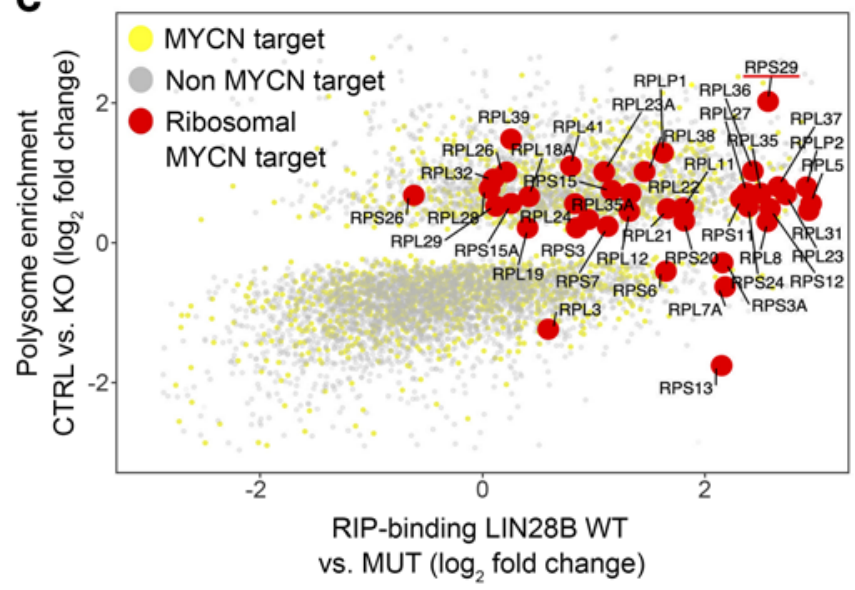

D
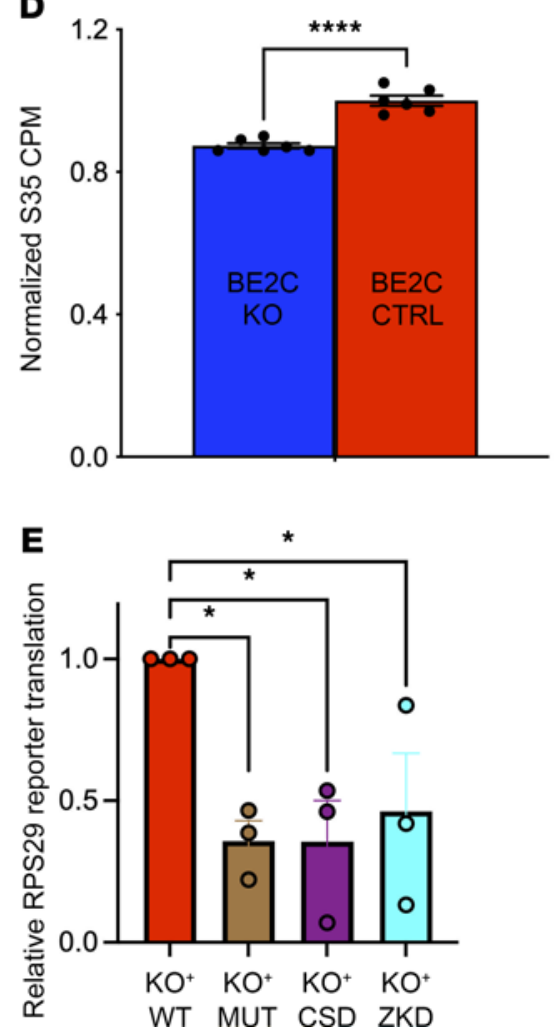

Figure 6. LIN28B cooperates with MYCN to alter ribosomal dynamics. (A) Venn diagrams of LIN28B-bound (gray) and transcripts with significant differential polysome enrichment (blue and red) as well as LIN28B-bound and transcriptional targets of MYCN (yellow). (B) Gene ontology analysis (GO Biological Process 2018) of indicated overlaps (LIN28B-bound and polysome-enriched transcripts [left] and LIN28B-bound and MYCN targets [right]). (C) Scatterplot depicting transcripts depending on their polysome enrichment and LIN28B binding. MYCN targets of the ribosome are indicated in red. (D) Protein synthesis analysis measured by metabolic labeling using S35-labeled methionine in the respective groups ( $n=6$ biological replicates per group). (E) In vitro translation assay with RPS29-reporter mRNA on lysates of BE2C-KO cells expressing the different LIN28B proteins: WT and deficient in RNA binding (MUT), only intact zinc knuckle domain (ZKD), and only intact cold shock domain (CSD) ( $n=3$ biological replicates). Differential comparisons were made between BE2C CTRL and BE2C LIN28B-KO cells. MYCN targets were defined using CHIPseeker (promoter $\leq 1 \mathrm{~kb}$ ). Statistical data in D were assessed using 2-tailed Student's $t$ test, in $\mathbf{E}$ using 1-way ANOVA with Tukey's multiple-comparison test; data are presented as mean $\pm \mathrm{SEM}$. ${ }^{*} P<0.05$; ${ }^{* * * *} P<0.0001$ $\mathrm{GO}$ analysis in B was performed with Enrichr. MYCN CHIP-Seq data were derived from Durbin et al. (74).

ribosomal proteins has been correlated with worse survival in different tumor types $(11,51)$, consistent with our observation of a LIN28B-associated ribosomal protein signature in stage 4 neuroblastoma. Additionally, we detected a strong correlation between $L I N 28 B$ and increased ribosomal signatures in other MYCNexpressing cancer types (Figure $7 \mathrm{H}$ ).
Previously, LIN28B-driven changes on polysome enrichment were mainly attributed to differential Argonaute (AGO) protein occupancy by let-7/mir-98 miRNAs in HEK293T cells (29). Our data showed that differential polysome enrichment due to LIN28B was independent of the let-7 miRNAs in a MYCN-amplified tumor model, highlighting differences in LIN28B translation programs in 
A

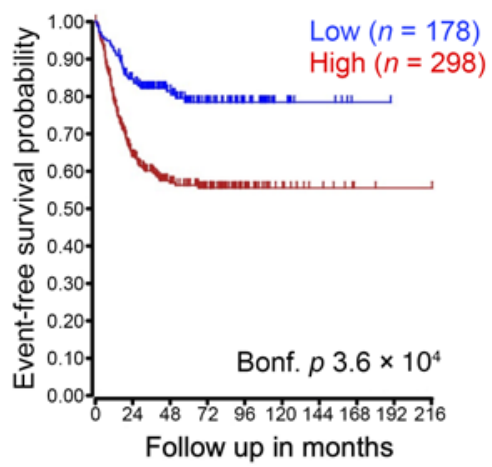

D

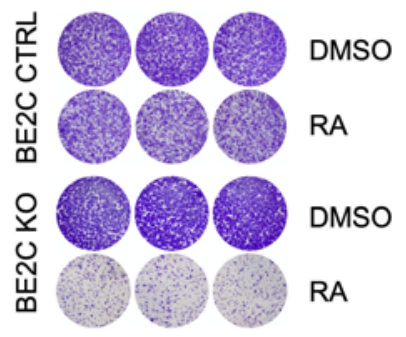

G $\quad 31$ TCGA datasets

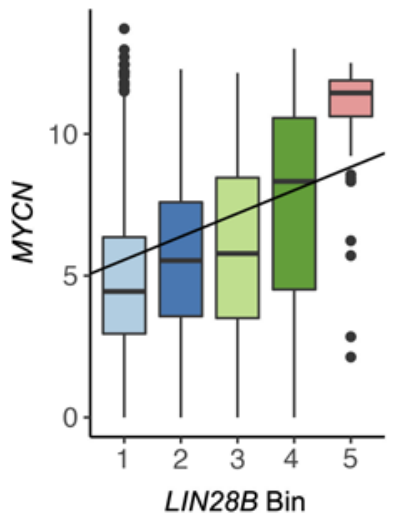

B

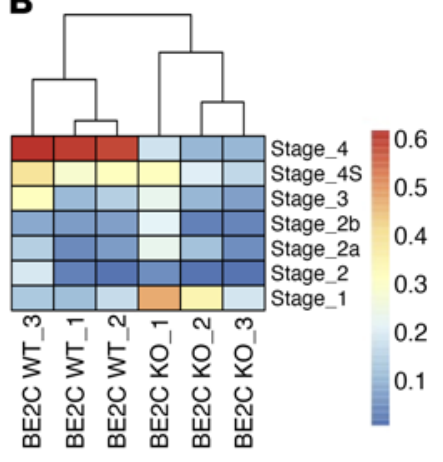

C

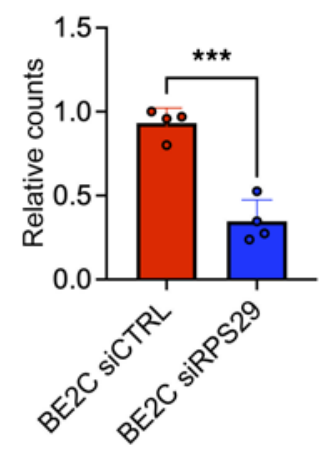

$\mathbf{E}$

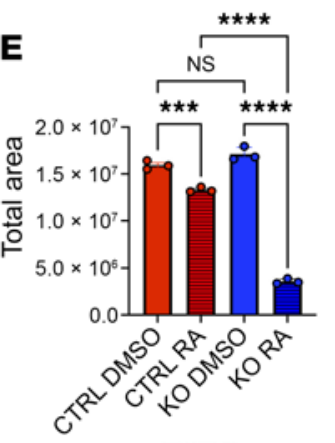

$\mathbf{F}$

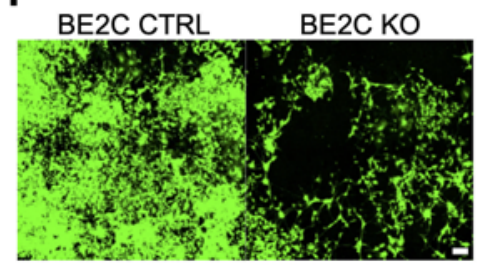

H

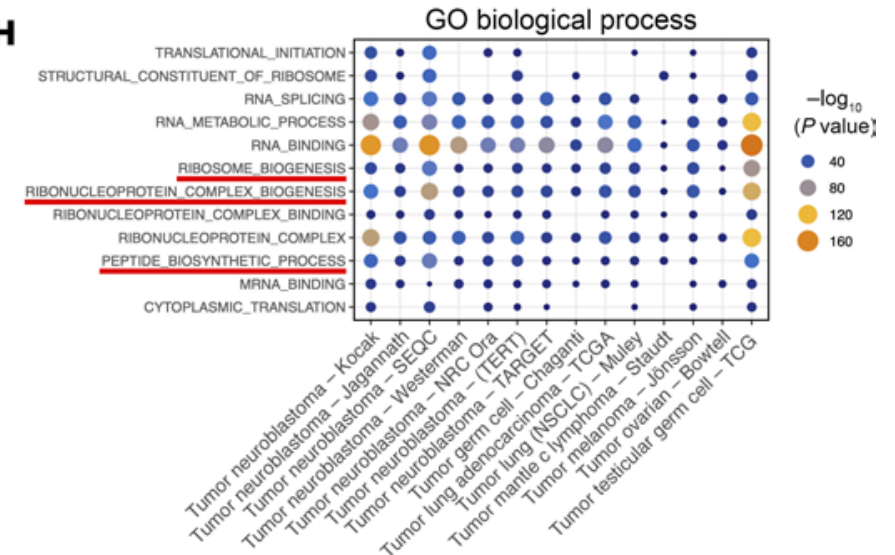

Figure 7. LIN28B expression defines stage 4 neuroblastoma and correlates with MYCN expression and ribosomal biogenesis. (A) Kaplan-Meier survival curve of the Kocak neuroblastoma data set depicting event-free survival for high and low LIN28B-expressing tumors. (B) Heatmap of BE2C WT and LIN28B-deficient cells clustering in the different stages of the Kocak study. (C) Relative counts of migrated BE2C cells upon transfection of siCTRL and siRNA targeting RPS29, 24 hours after plating ( $n=4$ biological replicates/group). (D) Representative crystal violet staining of BE2C CTRL and BE2C LIN28BKO cells after 7 days of treatment with DMSO and $2 \mu$ M retinoic acid. (E) Total area of BE2C cells shown in $\mathbf{D}$ assessed by Imagej ( $n=3$ biological replicates). (F) Representative fluorescence image of GFP-expressing BE2C CTRL (left) and BE2C LIN28B-KO cells (right) 10 days after treatment with $0.6 \mu M$ retinoic acid (see also Supplemental Video 1). (C) Box plots of the distribution of MYCN plotted as a function of LIN28B in 5 equal-sized bins in 31 TCCA RNA-Seq data sets. (H) Gene ontology analysis (GO Biological Process 2019) of genes positively correlated with LIN28B in the indicated studies. Statistical data in A were assessed using Bonferroni's test, in C using 2-tailed Student's $t$ test, and in $\mathbf{E}$ using 1-way ANOVA with Tukey's multiple-comparison test; data are presented as mean \pm SEM. ${ }^{* * *} P<0.001 ;{ }^{* * *} P<0.0001$. GO analysis in $\mathbf{A}$ and $\mathbf{H}$ were performed using the R2 database. Scale bar: $50 \mu \mathrm{m}(\mathbf{F})$.

different contexts. The regulatory mechanisms by which LIN28B alters translation of bound mRNAs are still not fully understood. One of the best-established binding targets of LIN28B is the let7 miRNA family. LIN28B's ZKD has been shown to bind to let-7 through the binding motif GGAG (52). Recently, the binding of the CSD on the stem loop region of let-7 was also shown to be essential for efficient inhibition of let-7 processing (21). Our in-depth analysis of polyribosome data sets in the context of LIN28B expression revealed distinct translational regulation in multiple cell types. Analysis of LIN28 mutant proteins lacking RNA binding capabilities for either the CSD or ZKD revealed the importance of the CSD as driver of polysome enrichment (53). Interestingly, none of the single mutants were capable of rescuing the invasive capabilities of BE2C neuroblastoma cells, indicating that, similar to its repressive function on let-7, both binding sites appear to be required to mediate the full effect of LIN28B in contributing to metastatic functions. 
Therapeutic targeting of the translational machinery in highly aggressive cancers has been previously suggested $(49,54,55)$, and various compounds that inhibit ribosomal biogenesis have shown therapeutic potential (4). In our model system of MYCN-driven disease, genetic inhibition of LIN28B showed strong synergistic effects with the clinically approved therapeutic retinoic acid in vitro, suggesting that tests of a combination of LIN28B inhibitors and retinoic acid is warranted in the clinical setting. The results of the differential binding of LIN28B's RNA binding sites could have important implications for the development of drugs targeting LIN28B's function in the context of advanced malignancies. Currently, development of LIN28 inhibitors mainly focuses on targeting the binding of the ZKD to the well-established GGAG motif (56). Our work suggests the CSD binding domain as an alternative for inhibition of LIN28B. In addition to mRNA and miRNAs, LIN28B directly interacts with a range of other proteins with RNA binding capabilities. Comparison of previously defined binding motifs for those proteins revealed preferential binding to AU-rich motifs. This suggests that LIN28B may cooperate with other RNA binding proteins to alter polysome enrichment of transcripts with similar AU-rich structure, a phenomenon that has previously been described for other RNA binding proteins as well (57). Most RNA binding proteins still have unknown motif specificity, but as motif discovery continues (57-59), future analyses are likely to uncover additional RNA binding proteins that collaborate with MYC/MYCN. These MYC/MYCN-supporting RNA binding proteins could be specifically targeted with the goal to revert metastatic translational outputs.

Invasion and dissemination are hallmarks of aggressive cancers. In this context, the processes of invasion and physiological tissue regeneration exhibit high similarities. LIN28 has previously been linked to enhanced tissue regeneration in a variety of adult tissues (60). LIN28 contributes to reprogramming of cellular metabolism with increased ATP production. Interestingly, loss of let-7 was not sufficient to fully phenocopy the LIN28-driven phenotype, implying at least a partially let-7-independent effect. It is tempting to speculate that LIN28 rewires polysome enrichment in this context as well.

Altogether, our work uncovered potentially new regulatory mechanisms by which LIN28B cooperates with MYCN to alter ribosomal dynamics. Our observations thus have important implications for MYCN-driven malignancy and potentially other MYCinduced tumors as well.

\section{Methods}

\section{Cell lines}

BE2C (ATCC, CRL-2268); SK-N-AS (ATCC, CRL-2137); SH-SY5Y (ATCC, CRL-2266); HEK293T (ATCC, 11268); CHP-212 (ATCC, CRL-2273); and Kelly cells (Sigma-Aldrich, 92110411-1VL) were maintained in a 1:1 mixture of Dulbecco's modified essential medium and Ham's F-12 medium (DMEM/F12) (except HEK293T, which were maintained in DMEM) with $10 \%$ inactivated FCS, $1 \mu \mathrm{g} / \mathrm{mL}$ penicillin, and $1 \mathrm{U} / \mathrm{mL}$ streptomycin. All cell lines were purchased for the purposes of this study, were not among commonly misidentified cell lines (per International Cell Line Authentication Committee; https://iclac. org), and tested negative for mycoplasma contamination.

\section{Generation of LIN28B (mutant) proteins}

FLAG-LIN28B (LIN28B WT), FLAG LIN28B RNA binding mutant (LIN28B MUT) (mutations in CSD and ZKD), and FLAG LIN28B single RNA binding site mutant transgenes (CSD - mutation in the ZKD; ZKD - mutations in the CSD) were generated by introducing mutations that have been shown to abrogate RNA binding in the highly conserved homologous regions of LIN28A: (a) CSD mutant, W36A/ F45A/F63A mutations; (b) ZND mutant, H137A/H159A mutations; and (c) CSD/ZND mutant harboring all 5 mutations $(52,53,61,62)$.

Mutant constructs were generated using the QuikChange SiteDirected Mutagenesis Kit, as per the manufacturer's guidelines (Agilent). The following primers were used: KT-hLIN28B-W36A_S, gAACTGGCCACTGTAAGGCGTTCAATGTGCGCATGG; KT-hLIN28B-W36A_AS, CCATGCGCACAT TGAACGCCT TACAGTGGCCAGT TC; KT-hLIN28B-F45A_S, TGTGCGCATGGGATTTGGAGCCATCTCCATGATAAACCGA; KT-hLIN28B-F45A_AS, TCGGTTTATCATGGAGATGGCTCCAAATCCCATGCGCACA; KT-hLIN28B-F63A_S, CCCTTGGATATTCCAGTCGATGTAGCTGTACACCAAAGCAAACTATT; KT-hLIN28B-F63A_AS, AATAGTTTGCTTTGGTGTACAGCTACATCGACTGGAATATCCAAGGG; KT-hLIN28B-H137A_S, TACAACTGTGGTGGCCTTGATGCTCATGCTAAGGAATGTAG;KT-hLIN28B-H137A_AS, CTACATTCCTTAGCATGAGCATCAAGGCCACCACAGTTGTA; KT-hLIN28B-H159A_S, ACTGTCAGAGCATCATGGCCATGGTGGCAAACTGCC; KT-hLIN28B-H159A_AS, GGCAGTTTGCCACCATGGCCATGATGCTCTGACAGT.

Constructs were cloned into the pFUGW plasmid - a gift from Feng Zhang (Addgene 52962; ref. 63).

\section{SiRNA transfections}

BE2C and Kelly cells were reverse transfected using Lipofectamine 2000 (Life Technologies) into 6-well plates using the appropriate siRNA. Cells were harvested 72 hours after for analysis by Western blot. Transwell assays were performed similarly, but 48 hours after transfection, cells were plated as described below. Control siRNA (Life Technologies, 439846) and RPS29 siRNA (Horizon Discovery, L-011157-000005) were obtained commercially.

\section{CRISPR/Cas9}

Cas9/gRNA-coexpressing lentiviral constructs (lentiCRISPRv2) were generated and lentiviral particles were produced as previously described $(63,64)$, using protocols and gRNA design tools from http://www. genome-engineering.org. Puromycin selection began 24 hours after lentiviral infection of BE2C and Kelly cells. Oligonucleotides used for gRNA cloning were as follows: LIN28B exon 2, CACCGCATCGACTGGAATATCCAAG and AAACCTTGGATATTCCAGTCGATGC; LIN28B exon 3.1, CACCGCAGAGCAAACTATTCATGGA and AAACTCCATGAATAGTTTGCTCTGC; CTRL1, CACCGGGACGCTAAACCAACGGTGCand AAACGCACCGTTGGTTTAGCGTCCC;CTRL2, CACCGTGAGGATCATGTCGAGCGCC and AAACGGCGCTCGACATGATCCTCAC.

\section{Cas9/gRNA constructs}

LentiCRISPRv2 was a gift from Feng Zhang (63) (Addgene plasmid 2961) and LentiCRISPRv2-mCherry was a gift from Agata Smogorzewska (Addgene plasmid 99154; http://n2t.net/addgene:99154).

\section{Lentiviral production}

To produce lentiviral particles, HEK293T cells were transfected with 
the modified pFUGW of interest and second-generation lentiviral packaging plasmid psPAX2 and VSV-G plasmid pMD2.G, using X-tremeGENE 9 transfection reagent (Sigma-Aldrich, 06365787 001) and Opti-MEM (Gibco, 31985070). The viral supernatant was collected at 24 and 48 hours after transfection, sterile filtered, and stored at $-80^{\circ} \mathrm{C}$. Firefly luciferase-F2A-GFP lentivirus was commercially obtained from Biosettia (GlowCell-16).

Next, $1 \times 10^{6}$ cells were infected for 8 hours with $0.5 \mathrm{~mL}$ of the viral supernatant in $8 \mu \mathrm{g} / \mu \mathrm{L}$ polybrene. Packing and envelope plasmids were gifts from Didier Trono (psPAX2, Addgene plasmid 12260; pMD2.G, Addgene plasmid 12259).

\section{Proliferation assays}

Cell proliferation was evaluated by using a CCK8 Kit (Sigma-Aldrich, 96992). First, $1 \times 10^{4}$ cells were plated per well in 96-well plates. After adding CCK8 reagent to each well, plates were incubated for 1 hour at $37^{\circ} \mathrm{C}$ and absorbance was measured at $490 \mathrm{~nm}$.

\section{Migration assays}

First, $2 \times 10^{4}$ cells were plated per well in a 24 -well plate to generate a confluent monolayer. After cell adherence, a straight scratch was created using a P2O0 pipette tip. To track wound closure, images were taken at different time points and the analyses were performed with Image $(\mathrm{NIH})$.

\section{Transwell assay}

Cells $\left(2 \times 10^{5}\right)$ were plated on $8-\mu$ m pore Transwell chambers (Corning) in media containing $2 \% \mathrm{FBS}$, whereas media in the bottom chamber contained $10 \% \mathrm{FBS}$. After an incubation time of 24 hours at $37^{\circ} \mathrm{C}$, cells that had not invaded through the membrane were washed off and the remaining cells were fixed ( $4 \%$ PFA) and stained $(0.005 \%$ crystal violet). The area of invaded cells was calculated by using ImageJ software.

\section{Anoikis assays}

GFP-positive cells $\left(1 \times 10^{4}\right)$ were plated per well in a 96-well ultra-lowattachment plate to assess spheroid formation. After incubating the cells at $37^{\circ} \mathrm{C}$ for 3 days, immunofluorescence pictures were taken with Cell Voyager 7000 (Yokogawa). The sphere area and sphere numbers were calculated by using Image J.

A soft agar assay was performed in growth media containing $0.35 \%$ noble agar, and $6 \times 10^{3}$ cells were plated per well on 24 -well plates. The colonies were fixed (4\% PFA) and stained (0.005\% crystal violet) after 3 weeks. The colony number was counted by using ImageJ.

\section{Retinoic acid treatment}

Retinoic acid (Sigma-Aldrich, R2625) was dissolved in DMSO according to the manufacturer's protocol and added at a final concentration as indicated once daily.

\section{Western blot assays}

Cell lysis for Western blotting was performed by using RIPA buffer (Pierce) containing $1 \times$ protease inhibitor cocktail (Pierce) and protein concentration was assessed by BCA Protein Assay (Pierce). Proteins were resolved in $8 \%$ to $15 \%$ SDS-PAGE gels and transferred to PVDF membranes (Bio-Rad). After a 60-minute blocking step with $5 \%$ milk in PBS, the membrane was probed with antibodies as recommended by the manufacturer. Fluorescence was detected with a LiCOR Odyssey.
All analyses were performed with LI-COR imaging software. See complete unedited blots in the supplemental material.

The following antibodies were used: Rb anti-LIN28B (Cell Signaling Technology, 4196) 1:1000; Rb anti- $\alpha / \beta$-tubulin (Cell Signaling Technology, 2148S) 1:1000; Rb anti- $\beta$-actin (Cell Signaling Technology, 8457) 1:1000; Rb anti-IGF2BP-1 (Cell Signaling Technology, 2852) 1:1000; Ms anti-FLAG M2 (Sigma-Aldrich, F1804) 1:2000; Ms anti-vinculin (Abcam, ab91459) 1:10,000; Rb anti-RPS29 (Invitrogen, PA5-41744) 1:1000; IRDye 680LT goat anti-rabbit IgG (LI-COR Biosciences, 926-68021) 1:25,000; and IRDye 800CW goat anti-mouse IgG (LI-COR Biosciences, 926-32210) 1:15,000.

\section{Flow cytometry}

For metastatic livers, single-cell dissociation of extracted livers was performed using $0.25 \%$ trypsin in $37^{\circ} \mathrm{C}$ for 30 minutes. After cells were recovered from metastatic livers or ascites, red cell lysis was performed using Red Blood Cell Lysis buffer (Roche, 11814389 001). DAPI was used for live/dead discrimination. Flow cytometric data were collected on an LSRFortessa (BD), and FlowJo 8.7 (BD) was used for data analysis.

\section{Immunofluorescence staining}

For immunofluorescence staining, approximately $1 \times 10^{4}$ cells were plated per well in 4-well chamber slides. After 24 hours, cells were fixed with 4\% PFA for 15 minutes and blocked for 20 minutes $(0.2 \%$ Triton X-100, 3\% BSA). Antibodies were diluted in 0.1\% Triton X-100, $1 \%$ BSA and were used as recommended by the manufacturer. Images were taken with a Zeiss LSM 700 laser scanning confocal microscope.

The following antibodies were used: Rb anti-LIN28B (Cell Signaling Technology, 4196) 1:400 dilution; rhodamine phalloidin (Invitrogen, R415), dilution of $0.5 \mu \mathrm{L}$ of the $400 \times$ stock solution in 200 $\mu \mathrm{L}$ of PBS for each coverslip; goat anti-rabbit IgG secondary antibody Alexa Fluor 488 (Invitrogen, A-11008), final dilution $4 \mu \mathrm{g} / \mathrm{mL}$; VECTASHIELD antifade mounting medium with DAPI (Vector Laboratories, $\mathrm{H}-1200)$.

\section{Animals}

Immunocompromised mice (NOD.Cg-Prkd scid $I l 2 \mathrm{rg}^{\text {tmlwjl }} / \mathrm{SzJ}$ [NSG]) were obtained from The Jackson Laboratory (stock no. 005557) and used for all the in vivo assays.

\section{Tail vein assay}

First, $100 \mu \mathrm{L}$ PBS containing $1 \times 10^{5}$ GFP-luciferase-positive cells were injected into the tail vein of 10-week-old male NSG mice. After injection of D-luciferin (Promega, P1043) into the tail vein, bioluminescence monitoring was performed using the IVIS Spectrum In Vivo Imaging System (PerkinElmer) to assess the total photon count (p/s/ $\mathrm{cm}^{2} / \mathrm{sr}$ ). Livers and primary tumors were explanted and imaged separately to assess organ-specific bioluminescent signal and fixed in formalin and embedded in paraffin for IHC.

For the limiting-dilution assay, freshly infected luciferaseexpressing BE2C cells were sorted for mCherry-expressing vectors of CTRL1 and EX 3.1 gRNA and injected into the tail vein of NSG mice in dilutions of 4000, 400, and 40 cells/injection. Five weeks after injection, liver colonization was assessed using IVIS bioluminescence traction of explanted livers. The frequency of tumor-initiating cells was assessed as previously described (65). 


\section{Kidney capsule xenografts}

Cells were injected under the left kidney capsule of 10-week-old male NSG mice. Briefly, $2.5 \times 10^{5}$ cells were resuspended in Matrigel and PBS in a 5:1 ratio (DPBS from Gibco, 14190250; and Matrigel from Corning, 15585729). Mice were anesthetized with isoflurane, shaved, and disinfected with iodopovidone and $70 \%$ ethanol. Using sterile techniques, a small incision was made on the left lateral side of the spine. The left kidney was mobilized and the cell/Matrigel suspension was injected right under the kidney capsule. The wound was closed using clips. In case a nephrectomy was performed, mice underwent surgery again 4 days after initial injection. After mobilizing the kidney, 2 absorbable ligatures were applied on renal vasculature and an incision was made in between. The freed left kidney was removed and the wound was closed using clips. Bioluminescence tracking was performed as described above to monitor tumor burden. Mice were euthanized after 3 to 5 weeks. Livers and primary tumors were explanted and imaged separately to assess organ-specific bioluminescent signal.

\section{PDX assays}

Luciferase-expressing PDX cell lines were gifts from St. Jude Hospital in Memphis, Tennessee, USA. Cells were injected into the adrenal gland and propagated using the xenograft technique as previously described (66). After euthanizing the mice, the primary tumors were dissociated and transduced with LentiCRISPRv2-mCherry-virus for 12 hours. A small fraction of cells was cultured in neurosphere media (DMEM/F12 [1:1], B27, Gibco, 17504044; 20 ng/mL EGF, SigmaAldrich, E5036; $20 \mathrm{ng} / \mathrm{mL}$ FGF-2, Sigma-Aldrich, SRP4037) for up to 72 hours and imaged for mCherry, yellow fluorescent protein with Cell Voyager 7000 (Yokogawa). Next, $1 \times 10^{6}$ cells of each genotype were reimplanted in the adrenal gland as previously described (66). Bioluminescence tracking was performed as described above to monitor tumor burden. Mice were euthanized after 3 to 5 weeks. Livers and primary tumors were explanted and imaged separately to assess organ-specific bioluminescent signal.

\section{qPCR}

Total RNA was isolated from cells using TRIzol reagent (Invitrogen, 15596018) and an RNeasy Plus Mini Kit (Qiagen, 74136). RNA concentration was assessed with Quant-iT Qubit RNA BR Assay Kit (Invitrogen, Q10210). For mRNA and miRNA analysis, first cDNA was synthesized with miScript II RT Kit (Qiagen, 218161) followed by qPCR with the miScript SYBR Green PCR Kit (Qiagen, 218076).

\section{Primers}

miScript microRNA primer assays used for let-7 and Hs_RNU6 miRNAs were obtained commercially (Qiagen, 218300): $h$ LIN28B-FW, CCTTGGATATTCCAGTCGATGTAT; $h L I N 28 B-R V$, TGACTCAAGGCCTTTGGAAG; $h$ GAPDH-FW, ACCCAGAAGACTGTGGATGG; hGAPDH-RV, TTCAGCTCAGGGATGACCTT.

For all expression analyses, relative expression was determined using the $\Delta \Delta \mathrm{C}_{\mathrm{T}}$ method, unless otherwise noted.

\section{RNA IP}

For RNA IP, cell lysis was performed in M2 buffer containing RNAse inhibitor (Promega, N2615). As recommended by the manufacturer, the lysate was then incubated with anti-FLAG M2 affinity gel beads (Sigma-Aldrich) for different FLAG-tagged LIN28B pulldown followed by wash steps with M2 buffer. RNA bound to the M2 affinity gel beads was then isolated using miRNeasy Mini Kit (Qiagen, 217004).

\section{Polysome profiling}

Polysome fractionations were performed as described previously (67). Briefly, four $15-\mathrm{cm}$ plates of $80 \%$ confluent BE2C cells were treated with cycloheximide, lysed, and layered onto $10 \%$ to $50 \%$ sucrose gradient tube and centrifuged at 222,228g (36,000 rpm) in a Beckman SW-41Ti rotor for 2.5 hours at $4^{\circ} \mathrm{C}$. Gradients were fractionated and monitored at absorbance $254 \mathrm{~nm}$ (Brandel). Collected fractions were then used for RNA extraction using TRIzol LS reagent (Invitrogen, 10296028) and RNeasy Plus Mini Kit (Qiagen, 74136).

\section{Affinity purification and mass spectrometry}

BE2C cells were harvested in cold PBS and immediately lysed in M2 lysis buffer (50 mM Tris- $\mathrm{HCl}$ pH 7.5, 150 mM NaCl, 1 mM EDTA, 1\% Triton $\mathrm{X}-100)$ containing $2 \times$ protease and phosphatase inhibitors (Pierce). Where applicable, the buffer was supplemented with 100 $\mu \mathrm{g} / \mathrm{mL}$ RNase A (Qiagen). FLAG-tagged LIN28B was purified using anti-FLAG M2 affinity gel following the manufacturer's specifications (Sigma-Aldrich). BE2C cells expressing GFP instead of FLAG-LIN28B were used as controls. Affinity-purified proteins were separated in a $4 \%$ to $20 \%$ polyacrylamide gel (Bio-Rad) and visualized using Bio-Safe Coomassie stain (Bio-Rad). Each lane was split into 4 pieces, which were excised, chopped into $1 \mathrm{~mm}^{3}$ cubes, and treated with DTT to reduce disulfide bonds and iodoacetamide to alkylate cysteines. In-gel digestion of the proteins was performed with trypsin. The resulting peptides were extracted from the gel and analyzed by liquid chromatography tandem mass spectrometry as described previously (68).

\section{In vitro transcription and translation}

mRNAs were made by in vitro transcription with T7 RNA polymerase as previously described (69). Briefly, PCR templates were made using primers to add a T7 promoter and polyadenylate tail, and transcription was performed using gel-extracted products. RNAs were purified by lithium chloride/EDTA precipitation. In vitro translation extracts were made as previously described $(69,70)$. Each translation reaction contained 200 ng of mRNA; $50 \%(\mathrm{v} / \mathrm{v})$ in vitro translation lysate; and buffer to make the final reaction with $0.84 \mathrm{mM}$ ATP, $0.21 \mathrm{mM} \mathrm{GTP}, 21 \mathrm{mM}$ creatine phosphate (Roche), $45 \mathrm{U} / \mathrm{mL}$ creatine phosphokinase (Roche), $10 \mathrm{mM}$ HEPES-KOH pH 7.6, 2 mM DTT, 8 mM amino acids (Promega), $255 \mu \mathrm{M}$ spermidine, $3 \mathrm{mM} \mathrm{Mg}(\mathrm{OAc})_{2}$, and $50 \mathrm{mM}$ KOAc. Translation reactions were incubated for 1 hour at $30^{\circ} \mathrm{C}$, and luciferase activity was assayed following the manufacturer's protocol (GeneCopoeia).

\section{Metabolic labeling}

Equal numbers of control and LIN28B-KO BE2C cells were plated overnight and then washed with PBS and incubated in methionineand cysteine-free DMEM (Gibco) medium for 2 hours. Cells were then incubated for 1 hour after supplementation with $\left[{ }^{35} \mathrm{~S}\right]$-methionine ( $\left[{ }^{35} \mathrm{~S}\right]-\mathrm{Met} ; 100 \mathrm{mCi} / \mathrm{mL}$; PerkinElmer), after which they were washed with PBS to eliminate free radiolabeled amino acids. Total protein lysates were collected and the concentration of the proteins was measured by using Bradford assay. To measure radiolabeled secreted proteins, cells were starved in methionine- and cysteine-free DMEM/ F12 medium (Gibco) for 1 hour, incubated for 1 hour with $\left.{ }^{[35} \mathrm{S}\right]-\mathrm{Met}$ containing DMEM/F12 medium, washed twice with PBS, and then 
supplemented with fresh DMEM/F12 medium (without additional $\left.{ }^{[35} \mathrm{S}\right]$-Met) and the medium was collected immediately (0 minutes, to measure background, i.e., free amino acids) or after 1, 4 , or 8 hours. For the quantitation of $\left.{ }^{35} \mathrm{~S}\right]$-Met-labeled proteins in the lysates or in the supernatant medium, $\left.{ }^{[5} \mathrm{S}\right]$-Met-labeled proteins were subjected to liquid scintillation analysis.

\section{RNA-Seq and bioinformatic analysis}

Polysome and RIP-Seq experiments. Total RNA from polysome profiling and RNA IP was selected for polyA-mRNA with the NEBNext Poly(A) mRNA Magnetic Isolation Module (New England Biolabs, E7490L). Paired-end libraries were prepared using the NEBNext Ultra II RNA Library Prep Kit for Illumina (New England Biolabs, E7770L) according to the manufacturer's protocol. Raw paired reads (FASTQ files) from both data sets were pseudoaligned to human gene annotations (hg38) using Kallisto (version 0.44.0; ref. 71). Kallisto output abundance files were then imported for analysis with DESeq2 (version 1.24.0; ref. 72). Polysome enrichments were determined as $\log _{2}$ (fold change) (adjusted $P$ value $\leq 0.05$ ) of polysome fractions normalized to their total input RNA and compared between WT and LIN28BKO cells by likelihood-ratio tests via DESeq2. For differential gene expression of WT versus LIN28B-KO cells, $\log _{2}$ (fold change (adjusted $P$ value $\leq 0.05$ ) values were compared using the default Wald test in DESeq2. For RIP-Seq, LIN28B-bound RNAs were determined by likelihood-ratio tests via DESeq2, where either WT, CSD, or ZKD LIN28B pulldowns were compared with full RNA binding mutant LIN28B (LIN28B MUT) pulldowns (all groups normalized to their respective inputs). A $\log _{2}$ (fold change) of 1 or greater (adjusted $P$ value $\leq 0.05$ ) for LIN28B WT was defined as bound targets. As LIN28B single mutants (CSD and ZKD) had lower genes at a cutoff $\log _{2}$ ( fold change) of 1 or greater (adjusted $P$ value $\leq 0.05$ ), $\log _{2}$ (fold change) was set to 0.5 or greater (adjusted $P$ value $\leq 0.05$ ) for the comparisons of LIN28B WT, CSD, and ZKD to obtain an overall higher overlap of biological processes. Comparisons between input RNA-Seq, polysome sequencing, and RIP-Seq were performed on gene-level expression of protein coding transcripts (Gencode genes included in "protein-coding transcript sequences") detected across all experiments. Nucleotide content $(\mathrm{G}+\mathrm{C}$ or $\mathrm{A}+\mathrm{U})$ was determined for the longest annotated transcript region for each gene (Ensemble Gene Annotation hg38, 3'-UTR, CDS and 5'-UTR). Let-7 gene targets were downloaded from TargetScanHuman version 7.2 (73) and "conserved targets" were considered. Polysome enrichment data for overexpression of LIN28B from a previous study (29) were shared by Gene Yeo. Predicted motifs and motifs logos were obtained (58). Raw RNA-Seq data (FASTQ), DESeq2 normalized gene count tables, and DESeq2 results generated for this project are deposited in NCBI's Gene Expression Omnibus database (GEO GSE158320).

MYC/MYCN CHIP-Seq data processing. CHIP-Seq bed files (BE2C MYCN CHIP-Seq; ref. 74; HEK293 MYC CHIP-Seq; ref. 36) were annotated with the R package ChIPseeker using the USC hg19 genome.

Bulk RNA-Seq. Total RNA was depleted for ribosomal RNA using the NEBNext rRNA Depletion Kit (Human/Mouse/Rat) (New England Biolabs, E6310X). Paired-end libraries were prepared using the NEBNext Ultra II RNA Library Prep Kit for Illumina (New England Biolabs, E7770L) according to the manufacturer's protocol. FASTQ files containing paired-end RNA-Seq reads were aligned with Tophat 2.0.12 against the UCSC hg38 reference genome using Bowtie 2.2.4 with default settings $(75,76)$. Gene-level counts were obtained using the subRead featureCounts program (v1.5.1) using the parameter “--primary" and gene models from the UCSC hg38 Illumina iGenomes annotation package (77). Read counts were normalized using size factors as available by the DESeq2 package (72). The DESeq2 package was also used for differential expression analysis. Pathway enrichment analysis was performed using GSEA $(78,79)$, and GO enrichment analysis was performed using Enrichr $(80,81)$. Customized scripts were used to visualize pathway enrichment results.

Random forest classification. We obtained transcriptome data from 2 of the Kocak (GEO GSE45547) and Wang (GEO GSE3960) studies. These data sets profile the transcriptome of over 1000 patients and contain metadata regarding the clinical stage of the tumors. For each study, we identified genes preferentially expressed in each stage and used these genes to train random forest classifiers for each stage of the disease. The training data was standardized before training the models. Then, we standardized our BE2C RNA-Seq data and classified our samples using our trained tumor stage-specific machine learning models. These analyses assigned a probability score indicative of the probability of indistinguishability of our samples to stage-specific transcriptome profiles.

\section{R2 database}

Human patient microarray and RNA-Seq data sets were obtained from the R2: microarray analysis and visualization platform (http://r2.amc. nl) and analyzed using GraphPad Prism software. Significance was determined as stated. We used the Kaplan-Meier Scan (KaplanScan) tool available in the R2 database to perform Kaplan-Meier analysis.

\section{Statistics}

All in vitro experiments were performed at least 3 times independently, unless otherwise noted. A $P$ value less than 0.05 was considered significant. Statistical tests used are identified in each figure legend and were assessed using GraphPad Prism.

\section{Study approval}

All animal care and procedures were reviewed and approved by the Boston Children's Hospital IACUC that is accredited by AAALAC under protocol 15-12-3071R.

\section{Author contributions}

PM conceived of the project with intellectual guidance from GQD. PM performed experiments, analyzed data, made figures, and wrote the manuscript with input from all the authors. BPM performed liver single-cell dissociations. ELDR, DD, and MMA performed the computational analysis on sequencing experiments. PM, DSP, MP, and RIG performed polysome sequencing experiments. DSP prepared RNA-Seq libraries. DKJ and CK performed flow cytometry. JP, JWF, and DTT planned and performed in vitro experiments of migration and invasion. KMT and JTP cloned mutant constructs and performed IP mass spectrometry. DF and ASYL planned and performed in vitro translational assays.

\section{Acknowledgments}

We thank all members of the Daley lab, especially Jihan Osborne, Areum Han, and Alena Yermalovich for comments and invaluable advice during the project and on the manuscript. We thank R. Tomaino at the Taplin Biological Mass Spectrometry Core for 
assistance with mass spectrometry. We thank the St. Jude Hospital in Memphis for sharing PDX lines. This work was funded in part by grants from the NIH National Institute of General Medical Sciences (NIGMS) grant RO1 GM107536, the Alex's Lemonade Stand Foundation, and the Milky Way Research Foundation to GQD. PM was supported by the German Cancer Aid. RIG was supported by an Outstanding Investigator Award (R35CA232115) from the National Cancer Institute of the NIH. MP was supported by the National Institute of Diabetes and Digestive and Kidney Diseases of the NIH (K01DK121861). KMT was supported by an HHMI International Student Research Fellowship and a Herchel Smith Graduate Fellowship. ASYL was supported by a grant from the
Pew Biomedical Scholars program. ELDR would like to thank the Coordination for the Improvement of Higher Education Personnel (CAPES/Brazil) for fellowship support. DKJ was supported by the Leukemia and Lymphoma Society (TRP grant 6546-18). DSP was supported by award number T32GM007753 from the NIGMS. The content is solely the responsibility of the authors and does not necessarily represent the official views of the NIGMS or the NIH. The graphical abstract was created with BioRender.com.

Address correspondence to: George Q Daley, 300 Longwood Ave, Karp Family Building, 5th Floor, Boston, Massachusetts 02115, USA. Phone: 617.919.2015; Email: George.Daley@childrens.harvard.edu.
1. Kong J, Lasko P. Translational control in cellular and developmental processes. Nat Rev Genet. 2012;13(6):383-394.

2. Gebauer F, Hentze MW. Molecular mechanisms of translational control. Nat Rev Mol Cell Biol. 2004;5(10):827-835.

3. Holcik M, Sonenberg N. Translational control in stress and apoptosis. Nat Rev Mol Cell Biol. 2005;6(4):318-327.

4. Pelletier J, et al. Ribosome biogenesis in cancer: new players and therapeutic avenues. Nat Rev Cancer. 2018;18(1):51-63.

5. Barna M, et al. Suppression of Myc oncogenic activity by ribosomal protein haploinsufficiency. Nature. 2008;456(7224):971-975.

6 . Boon $\mathrm{K}$, et al. $\mathrm{N}$-myc enhances the expression of a large set of genes functioning in ribosome biogenesis and protein synthesis. EMBOJ. 2001;20(6):1383-1393.

7. van Riggelen J, et al. MYC as a regulator of ribosome biogenesis and protein synthesis. Nat Rev Cancer. 2010;10(4):301-309.

8. Ruggero D. The role of Myc-induced protein synthesis in cancer. Cancer Res. 2009;69(23):8839-8843.

9. Cho H, et al. RapidCaP, a novel GEM model for metastatic prostate cancer analysis and therapy, reveals myc as a driver of Pten-mutant metastasis. Cancer Discov. 2014;4(3):318-333.

10. Nowak DG, et al. MYC drives Pten/Trp53-deficient proliferation and metastasis due to IL6 secretion and AKT suppression via PHLPP2. Cancer Discov. 2015;5(6):636-651.

11. Ebright RY, et al. Deregulation of ribosomal protein expression and translation promotes breast cancer metastasis. Science. 2020;367(6485):1468-1473.

12. Ambros V, Horvitz H. Heterochronic mutants of the nematode Caenorhabditis elegans. Science. 1984;226(4673):409-416.

13. Viswanathan SR, et al. Lin28 promotes transformation and is associated with advanced human malignancies. Nat Genet. 2009;41(7):843-848.

14. Nguyen LH, et al. Lin $28 \mathrm{~b}$ is sufficient to drive liver cancer and necessary for its maintenance in murine models. Cancer Cell. 2014;26(2):248-261.

15. Molenaar JJ, et al. LIN28B induces neuroblastoma and enhances MYCN levels via let-7 suppression. Nat Genet. 2012;44(11):1199-1206.

16. Diskin SJ, et al. Common variation at $6 \mathrm{q} 16$ within HACE1 and LIN28B influences susceptibility to neuroblastoma. Nat Genet. 2012;44(10):1126-1130.

17. Madison BB, et al. LIN28B promotes growth and tumorigenesis of the intestinal epithelium via
Let-7. Genes Dev. 2013;27(20):2233-2245.

18. Urbach A, et al. Lin 28 sustains early renal progenitors and induces Wilms tumor. Genes Dev. 2014;28(9):971-982.

19. Tu HC,et al. LIN28 cooperates with WNT signaling to drive invasive intestinal and colorectal adenocarcinoma in mice and humans. Genes Dev. 2015;29(10):1074-1086.

20. Viswanathan SR, et al. Selective blockade of microRNA processing by Lin28. Science. 2008;320(5872):97-100.

21. Ustianenko D, et al. LIN28 selectively modulates a subclass of let-7 microRNAs. Mol Cell. 2018;71(2):271-283.

22. Sampson VB, et al. MicroRNA let-7a down-regulates MYC and reverts MYC-induced growth in Burkitt lymphoma cells. Cancer Res. 2007;67(20):9762-9770.

23. Johnson SM, et al. RAS is regulated by the let-7 microRNA family. Cell. 2005;120(5):635-647.

24. Mayr C, et al. Disrupting the pairing between let-7 and Hmga 2 enhances oncogenic transformation. Science. 2007;315(5818):1576-1579.

25. Cho J, et al. LIN28A is a suppressor of ER-associated translation in embryonic stem cells. Cell. 2012;151(4):765-777.

26. Wilbert ML, et al. LIN28 binds messenger RNAs at GGAGA motifs and regulates splicing factor abundance. Mol Cell. 2012;48(2):195-206.

27. Graf R, et al. Identification of LIN28B-bound mRNAs reveals features of target recognition and regulation. RNA Biol. 2013;10(7):1146-1159.

28. Hafner M, et al. Identification of mRNAs bound and regulated by human LIN28 proteins and molecular requirements for RNA recognition. RNA. 2013;19(5):613-626

29. Tan FE, et al. A transcriptome-wide translational program defined by LIN28B expression level. Mol Cell. 2019;73(2):304-313.

30. Beckers A, et al. MYCN-driven regulatory mechanisms controlling LIN28B in neuroblastoma. Cancer Lett. 2015;366(1):123-132.

31. Chang TC, et al. Lin-28B transactivation is necessary for Myc-mediated let-7 repression and proliferation. Proc Natl Acad Sci US A. 2009;106(9):3384-3389.

32. Dangi-Garimella S, et al. Raf kinase inhibitory protein suppresses a metastasis signalling cascade involving LIN28 and let-7. EMBO J. 2009;28(4):347-358.

33. Jiang $X$, et al. Blockade of miR-150 maturation by MLL-fusion/MYC/LIN-28 is required for MLL-associated leukemia. Cancer Cell. 2012;22(4):524-535.

34. Powers JT, et al. Multiple mechanisms disrupt the let-7 microRNA family in neuroblastoma. Nature. 2016;535(7611):246-251.

35. Braekeveldt N, et al. Patient-derived xenograft models reveal intratumor heterogeneity and temporal stability in neuroblastoma. Cancer Res. 2018;78(20):5958-5969.

36. Thomas LR, et al. Interaction with WDR5 promotes target gene recognition and tumorigenesis by MYC. Mol Cell. 2015;58(3):440-452.

37. Kocak H, et al. Hox-C9 activates the intrinsic pathway of apoptosis and is associated with spontaneous regression in neuroblastoma. Cell Death Dis. 2013;4(4):e586-e586.

38. Wang Q, et al. Integrative genomics identifies distinct molecular classes of neuroblastoma and shows that multiple genes are targeted by regional alterations in DNA copy number. Cancer Res. 2006;66(12):6050-6062.

39. Matthay KK, et al. Neuroblastoma. Nat Rev Dis Primers. 2016;2:16078.

40. Matthay KK, et al. Treatment of high-risk neuroblastoma with intensive chemotherapy, radiotherapy, autologous bone marrow transplantation, and 13-cis-retinoic acid. Children's Cancer Group. N Engl JMed. 1999;341(16):1165-1173.

41. Franses JW, et al. Pancreatic circulating tumor cell profiling identifies LIN28B as a metastasis driver and drug target. Nat Commun. 2020;11(1):3303.

42. Tang XH, Gudas LJ. Retinoids, retinoic acid receptors, and cancer. Annu Rev Pathol. 2011;6(1):345-364.

43. Adhikary S, Eilers M. Transcriptional regulation and transformation by Myc proteins. Nat Rev Mol Cell Biol. 2005;6(8):635-645.

44. Malynn BA, et al. N-myc can functionally replace c-myc in murine development, cellular growth, and differentiation. Genes Dev. 2000;14(11):1390-1399.

45. Rajasekhar VK, et al. Oncogenic Ras and Akt signaling contribute to glioblastoma formation by differential recruitment of existing mRNAs to polysomes. Mol Cell. 2003;12(4):889-901.

46. Hsieh AC, et al. The translational landscape of mTOR signalling steers cancer initiation and metastasis. Nature. 2012;485(7396):55-61.

47. Robichaud N, et al. Phosphorylation of eIF4E promotes EMT and metastasis via translational control of SNAIL and MMP-3. Oncogene. 
2015;34(16):2032-2042.

48. Robichaud N, et al. Translational control in cancer. Cold Spring Harb Perspect Biol. 2019;11(7):a032896

49. Truitt ML, Ruggero D. New frontiers in translational control of the cancer genome. Nat Rev Cancer. 2016;16(5):288-304.

50. Prakash V, et al. Ribosome biogenesis during cell cycle arrest fuels EMT in development and disease. Nat Commun. 2019;10(1):2110.

51. Luo S, et al. Highly expressed ribosomal protein L34 indicates poor prognosis in osteosarcoma and its knockdown suppresses osteosarcoma proliferation probably through translational control. Sci Rep. 2016;6(1):37690.

52. Nam Y, et al. Molecular basis for interaction of let-7 microRNAs with Lin28. Cell. 2011;147(5):1080-1091.

53. Balzer E, Moss EG. Localization of the developmental timing regulator Lin28 to mRNP complexes, P-bodies and stress granules. RNA Biol. 2007;4(1):16-25.

54. Bhat $\mathrm{M}$, et al. Targeting the translation machinery in cancer. Nat Rev Drug Discov. 2015;14(4):261-278.

55. Devlin JR, et al. Combination therapy targeting ribosome biogenesis and mRNA translation synergistically extends survival in MYC-driven lymphoma. Cancer Discov. 2016;6(1):59-70.

56. Wang L, et al. Small-molecule inhibitors disrupt let-7 oligouridylation and release the selective blockade of let-7 processing by LIN28. Cell Rep. 2018;23(10):3091-3101.

57. Dominguez D, et al. Sequence, structure, and context preferences of human RNA binding proteins. Mol Cell. 2018;70(5):854-867.

58. Ray D, et al. A compendium of RNA-binding motifs for decoding gene regulation. Nature. 2013;499(7457):172-177.

59. Jolma A, et al. Binding specificities of human
RNA-binding proteins toward structured and linear RNA sequences. Genome Res. 2020;30(7):962-973.

60. Shyh-Chang N, et al. Lin28 enhances tissue repair by reprogramming cellular metabolism. Cell. 2013;155(4):778-792.

61. Heo I, et al. Lin28 mediates the terminal uridylation of let-7 precursor MicroRNA. Mol Cell. 2008;32(2):276-284.

62. Piskounova E, et al. Determinants of microRNA processing inhibition by the developmentally regulated RNA-binding protein Lin28. J Biol Chem. 2008;283(31):21310-21314.

63. Sanjana NE, et al. Improved vectors and genomewide libraries for CRISPR screening. Nat Methods. 2014;11(8):783-784.

64. Shalem O, et al. Genome-scale CRISPR-Cas9 knockout screening in human cells. Science. 2014;343(6166):84-87.

65. Hu Y, Smyth GK. ELDA: extreme limiting dilution analysis for comparing depleted and enriched populations in stem cell and other assays. JImmunol Methods. 2009;347(1-2):70-78.

66. Stewart E, et al. Orthotopic patient-derived xenografts of paediatric solid tumours. Nature. 2017;549(7670):96-100.

67. Lin S, et al. The m(6)A methyltransferase METTL3 promotes translation in human cancer cells. Mol Cell. 2016;62(3):335-345.

68. Haas W, et al. Optimization and use of peptide mass measurement accuracy in shotgun proteomics. Mol Cell Proteomics. 2006;5(7):1326-1337.

69. Lee ASY, et al. eIF3 targets cell-proliferation messenger RNAs for translational activation or repression. Nature. 2015;522(7554):111-114.

70. Rakotondrafara AM, Hentze MW. An efficient factor-depleted mammalian in vitro translation system. Nat Protoc. 2011;6(5):563-571.

71. Bray NL, et al. Near-optimal probabilistic RNA-seq quantification. Nat Biotechnol.
2016;34(5):525-527.

72. Love MI, et al. Moderated estimation of fold change and dispersion for RNA-seq data with DESeq2. Genome Biol. 2014;15(12):550.

73. Agarwal V, et al. Predicting effective microRNA target sites in mammalian mRNAs. Elife. 2015;4:e05005.

74. Durbin AD, et al. Selective gene dependencies in MYCN-amplified neuroblastoma include the core transcriptional regulatory circuitry. Nat Genet. 2018;50(9):1240-1246.

75. Kim D, et al. TopHat2: accurate alignment of transcriptomes in the presence of insertions, deletions and gene fusions. Genome Biol. 2013;14(4):R36

76. Langmead B, Salzberg SL. Fast gappedread alignment with Bowtie 2. Nat Methods. 2012;9(4):357-359.

77. Liao Y, et al. featureCounts: an efficient general purpose program for assigning sequence reads to genomic features. Bioinformatics. 2014;30(7):923-930.

78. Subramanian A, et al. Gene set enrichment analysis: a knowledge-based approach for interpreting genome-wide expression profiles. Proc Natl Acad SciUS A. 2005;102(43):15545-15550.

79. Mootha VK, et al. PGC-1alpha-responsive genes involved in oxidative phosphorylation are coordinately downregulated in human diabetes. Nat Genet. 2003;34(3):267-273.

80. Chen EY, et al. Enrichr: interactive and collaborative HTML5 gene list enrichment analysis tool. BMC Bioinformatics. 2013;14(1):128.

81. Kuleshov MV, et al. Enrichr: a comprehensive gene set enrichment analysis web server 2016 update. Nucleic Acids Res. 2016;44(w1):W90-W97.

82. Dehairs J, et al. CRISP-ID: decoding CRISPR mediated indels by Sanger sequencing. Sci Rep. 2016;6(1):28973. 